\title{
Experimental results on electron beam combination and bunch frequency multiplication
}

\author{
Roberto Corsini, ${ }^{1}$ Arnaud Ferrari, ${ }^{2}$ Louis Rinolfi, ${ }^{1}$ Philippe Royer, ${ }^{1}$ and Frank Tecker ${ }^{1}, *$ \\ ${ }^{1}$ CERN, Geneva, Switzerland \\ ${ }^{2}$ Department of Radiation Sciences, Uppsala University, Uppsala, Sweden
}

(Received 4 December 2003; published 30 April 2004)

\begin{abstract}
The aim of the CLIC Test Facility CTF3 at CERN is to demonstrate the feasibility of the key points of the two-beam acceleration based compact linear collider study. In particular, it addresses the efficient generation of a drive beam with the appropriate time structure of the electron bunches in order to produce high power rf pulses at a frequency of $30 \mathrm{GHz}$. This time structure requires a high bunch repetition frequency. It is obtained by successive injections of bunch trains into an isochronous ring using transversely deflecting rf structures. The major goal of the now completed first phase of the CTF3 was to achieve the bunch train combination at low charge. In this paper, we give a description of the project and summarize the experimental results, with a focus on the successful bunch frequency multiplication for various factors up to 5 .
\end{abstract}

DOI: 10.1103/PhysRevSTAB.7.040101

PACS numbers: 29.17.+w, 29.27.Eg, 41.85.Ja, 41.85.Ct

\section{INTRODUCTION}

The compact linear collider (CLIC) study [1] aims at a multi- $\mathrm{TeV}(0.5-5 \mathrm{TeV}$ center-of-mass energy), high-luminosity $\left(2-8 \times 10^{34} \mathrm{~cm}^{-2} \mathrm{~s}^{-1}\right)$ electron-positron collider for particle physics. The CLIC scheme is based on a two-beam acceleration concept where a high-frequency $(30 \mathrm{GHz})$ high-gradient $(150 \mathrm{MV} / \mathrm{m})$ linear accelerator is powered by a low-energy $(2.1 \mathrm{GeV})$, high-intensity drive beam running parallel to the main beam.

One major challenge of the CLIC two-beam acceleration scheme is the generation of the drive beam electron pulses with the required high-current $(150 \mathrm{~A})$ and highfrequency bunch structures needed for $30 \mathrm{GHz}$ rf power production. The required drive beam cannot be obtained directly from an electron source with the present technology, in particular, in terms of bunch repetition frequency and beam pulse length. Furthermore, a highly efficient drive beam acceleration is required.

Therefore, a long electron pulse is accelerated by lowfrequency $(937 \mathrm{MHz})$, normal conducting traveling-wave cavities, working with strong beam loading. The structures are relatively short to minimize rf losses in the copper. Because of the strong beam loading, $97 \%$ of the power is given to the electron beam, with virtually no power sent to rf loads. The long drive beam pulse is then subdivided into subpulses by means of transverse rf deflectors, working at half the bunch repetition frequency. The subpulses are then recombined in stages, multiplying the current and the bunch frequency at the same time. The main manipulation for bunch frequency multiplication consists of sending the beam through an isochronous combiner ring using rf deflectors to inject and combine the electron bunch trains.

*Electronic address: Frank.Tecker@cern.ch
The aim of the CLIC Test Facility 3 (CTF3) project is to demonstrate the technical feasibility of the key concepts of CLIC. CTF3 is scaled down from CLIC, working at a frequency of $3 \mathrm{GHz}$ with lower beam current (35 A) and at lower energy (180 MeV). CTF3 will also provide a $30 \mathrm{GHz}$ rf source with the CLIC nominal peak power and pulse length for component tests. It is being built in stages over several years from 2001 to 2007.

A low current test of the bunch train combination was performed during 2001/02 in the first phase of CTF3 (the so-called Preliminary Phase), where the injection by rf deflectors into an isochronous ring and the multiplication of the bunch repetition frequency were demonstrated with short pulses. This paper describes the Preliminary Phase of CTF3 and presents the results of the experiments that were performed.

\section{DESCRIPTION OF THE CTF3 PRELIMINARY PHASE}

The Preliminary Phase of CTF3 made maximum use of the existing hardware of the former LEP Pre-Injector (LPI) complex [2] at CERN, composed of a $3 \mathrm{GHz}$ linear accelerator and an accumulator ring. The aim of the CTF3 Preliminary Phase was to accelerate several electron pulses in the linac and combine them in the ring using the injection scheme with rf deflectors described in detail later. Some major modifications had to be performed in order to adapt the former LPI installation to the requirements of CTF3. In particular, a new thermionic gun allowing a multipulse operation was designed and built by LAL-Orsay [3]. A matching section was included at the end of the linac, and both the injection line and the ring were modified to be isochronous. Two rf deflectors were installed in the injection region of the ring. The general layout of the facility is shown in Fig. 1 and a more detailed description can be found in Ref. [4]. The modifications were performed between April and September 


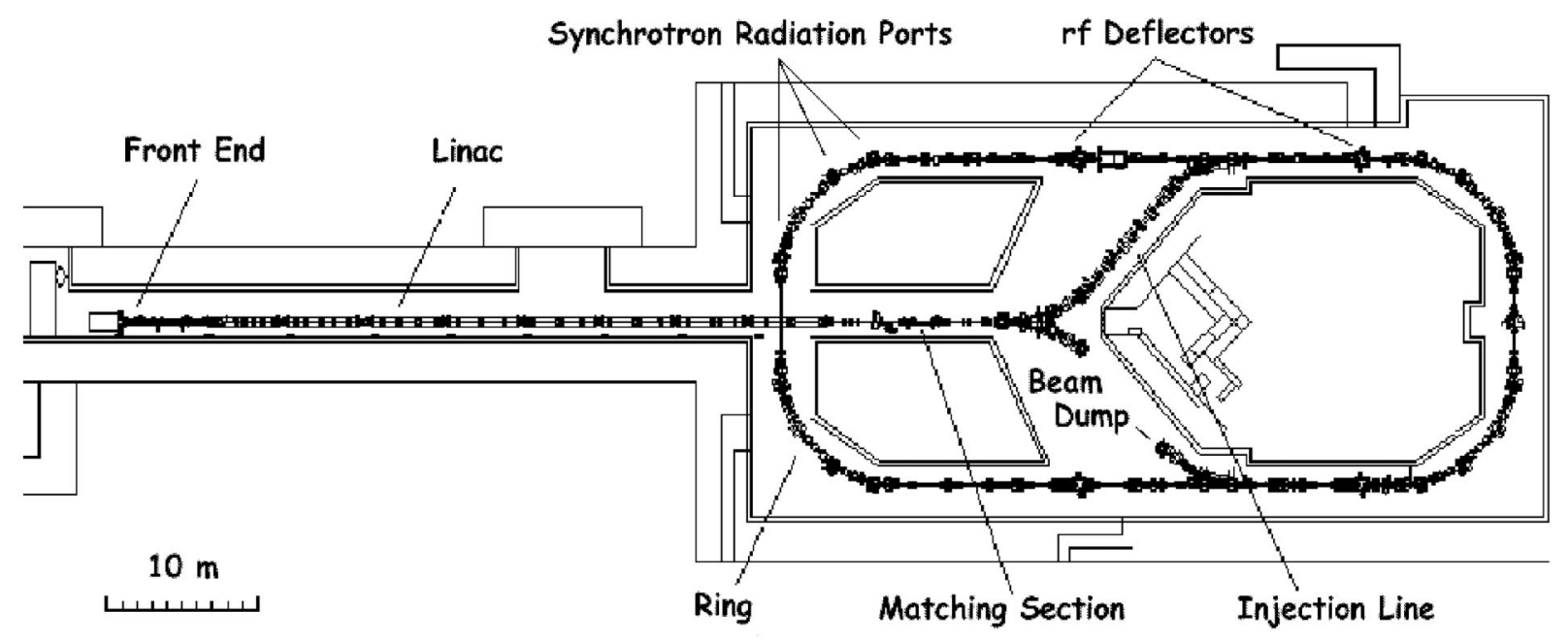

FIG. 1. General layout of the CTF3 Preliminary Phase.

2001, after which the CTF3 Preliminary Phase was commissioned and operated between September 2001 and October 2002.

\section{A. Layout and beam structure}

The front end of the linac consisted of a thermionic gun, a single-cell prebuncher, and a standing wave buncher structure. The thermionic gun operated at $90 \mathrm{kV}$ and delivered a train of up to seven electron pulses with an adjustable length between 2 and 10 ns FWHM, with a repetition frequency up to $50 \mathrm{~Hz}$. The pulses were spaced by $420 \mathrm{~ns}$, corresponding to the revolution period in the ring, as needed for the bunch frequency multiplication process. The $3 \mathrm{GHz}$ bunching system brought these pulses to an energy of about $5 \mathrm{MeV}$ and subdivided them into bunches spaced by $333 \mathrm{ps}$. For the nominal pulse length of $6.6 \mathrm{~ns}$ FWHM, there were approximately 20 bunches per pulse with a charge per bunch of about $0.1 \mathrm{nC}$, corresponding to a current of $0.3 \mathrm{~A}$. This charge was chosen in order to limit the beam loading in the accelerating structures, which were reused from LPI and not adapted to the high-current operation.

The linac was made of eight $3 \mathrm{GHz}$ traveling-wave accelerating structures, powered in groups of four by two $45 \mathrm{MW}$ klystrons. The whole electron pulse train $(2.5 \mu \mathrm{s})$ was accelerated within one klystron rf pulse of up to $4.5 \mu \mathrm{s}$, taking into account the filling time of the structures $(1.5 \mu \mathrm{s})$. The design energy at the end of the linac was $350 \mathrm{MeV}$.

A matching section of five quadrupole magnets was located at the end of the linac to match the transverse Twiss parameters of the beam to the injection line optics. A spectrometer line was also located in the same area, in order to measure the energy and the energy spread.

The injection line linked the linac to the ring. The ring circumference $C$ has to fulfil the condition

$$
C=\beta \lambda_{0}\left(n \pm \frac{1}{N}\right)
$$

where $N$ is the combination factor, $\beta$ the relativistic beta $\beta=v / c, \lambda_{0}$ the $\mathrm{rf}$ wavelength in the linac and in the deflectors, and $n$ an integer. With a given ring circumference, the frequency can be slightly detuned in order to switch between different combination factors. The nominal rf frequency is $2.99855 \mathrm{GHz}$ corresponding to a wavelength $\lambda_{0}=0.099979 \mathrm{~m}$.

A circumference $C=125.647 \mathrm{~m}$ was chosen, as this was the average length for combination factors 3 and 5 closest to the existing ring circumference [with $n=1257$ and a minus sign in Eq. (1)]. Thus, the existing accumulator ring had to be shortened by $17 \mathrm{~mm}$ by realigning the elements.

Synchrotron radiation ports were located next to three of the four bending magnets of one ring arc. At these different sources, the dispersion function ranges from zero to almost maximum value. The observation of the emitted light with a streak camera allowed a direct measurement of the time structure of the electron beam. This was the main instrument used for the demonstration of the bunch train combination process.

An extraction line was located at the opposite side of the injection region. A kicker magnet was used to deflect the beam into the extraction septum and send it into a beam dump.

\section{B. Bunch train combination by rf deflectors}

For the bunch train combination, the injection into the ring is performed using two horizontally deflecting rf structures. They are located in the ring with a horizontal betatron phase advance of $\pi$ between them to create a time-dependent local closed bump of the reference orbit. 

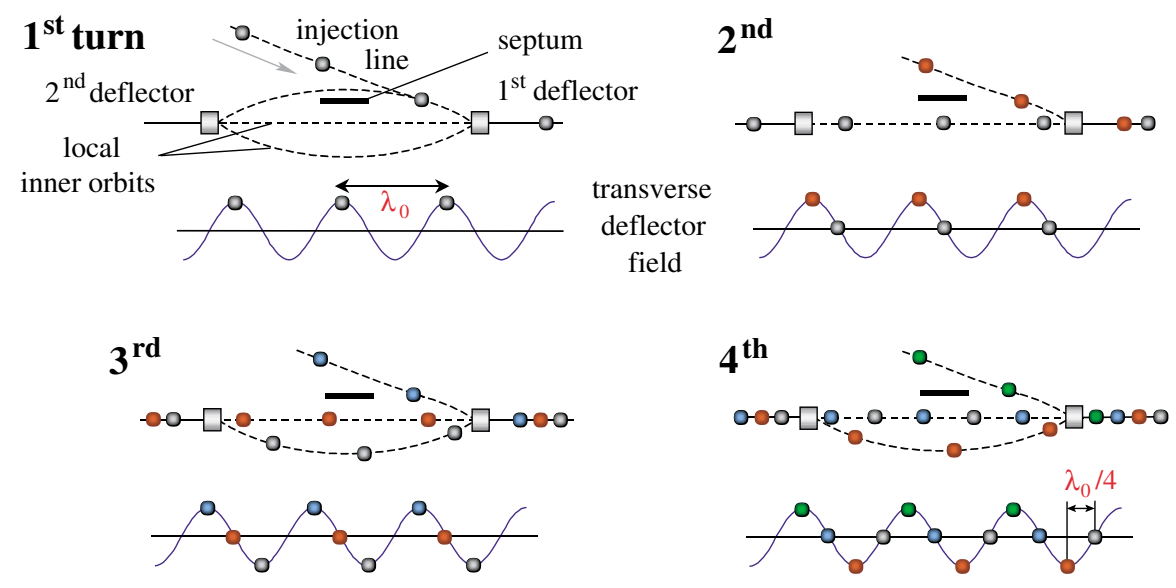

field

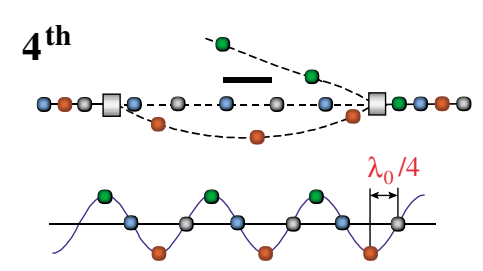

FIG. 2. (Color) Bunch train combination by injection with rf deflectors for a multiplication factor 4 . The images show the injection region of the ring for four successive turns of injected bunches and the corresponding bunch distribution on the rf field of the deflectors.

The deflection varies rapidly with time, allowing the interleaving of the bunches in the ring. The combination is possible for various combination factors. CLIC is based on two stages with a factor 4 each, while CTF3 with nominal current has a factor 5 in the ring. For didactic reasons, the principle of the injection with rf deflectors is explained in the following for a frequency multiplication factor 4 and shown in Fig. 2.

(1) The bunches of the incoming train always receive the maximum kick from the rf deflector and are deviated onto the closed orbit in the ring.

(2) With the condition of Eq. (1) fulfilled (for the combination factor $N=4$ ), the bunches pass the deflectors after one turn at the zero crossing of the rf field and stay on the unperturbed closed orbit. The second train is injected into the ring.

(3) After a second turn, the first-train bunches are kicked in the opposite direction and follow a closed bump between the deflectors, the second-train bunches arrive at the zero crossing, and the third train is injected.

(4) After the third turn, the first-train bunches arrive again at the zero crossing, the second-train bunches are kicked away from the septum, the third-train bunches are also at the zero crossing, and the fourth train is injected. The four trains are now combined into one single train and the initial bunch spacing is reduced by a factor 4 .

For combination factors other than 4 , the phase of the deflecting field at the passage of the bunches and hence the trajectories between the two rf deflectors change accordingly (see Fig. 3 for a combination factor 5).

The rf deflectors are short resonant, traveling-wave, iris-loaded structures with a negative group velocity. In order to obtain the nominal deflecting angle of $4.5 \mathrm{mrad}$ for injection with a beam energy of $350 \mathrm{MeV} / c$, a power of about $7 \mathrm{MW}$ is needed in each of the deflectors. They are powered by a common klystron with a phase shifter and a variable attenuator in one of the rf-network branches in order to allow relative phase and amplitude adjustments.

The first bunch train combination experiments were performed with already existing rf deflectors built by CERN. They were later replaced by newly designed structures, with a bigger iris aperture $(43 \mathrm{~mm}$ instead of $23 \mathrm{~mm}$ ). The latter were built by INFN-Frascati [5] and will be reused in a later phase of CTF3.

As a consequence of the rapid change of the deflecting field inside the deflectors, not only do the bunches on different turns experience different deflections but also the head and the tail of individual bunches are deflected more or less, as shown in Fig. 4.

This enlarges the transverse size of the circulating beam in the region between the two rf deflectors and represents the main contribution to the beam size at the septum location. Figure 5 shows the envelope of Gaussian bunches in the injection region.

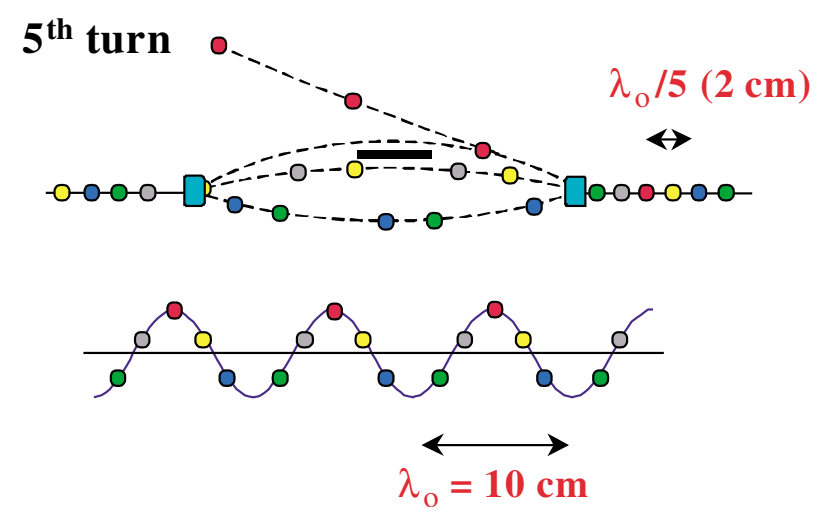

FIG. 3. (Color) Fifth passage of the first injected bunch train in the injection region for a combination with a multiplication factor of 5 . 


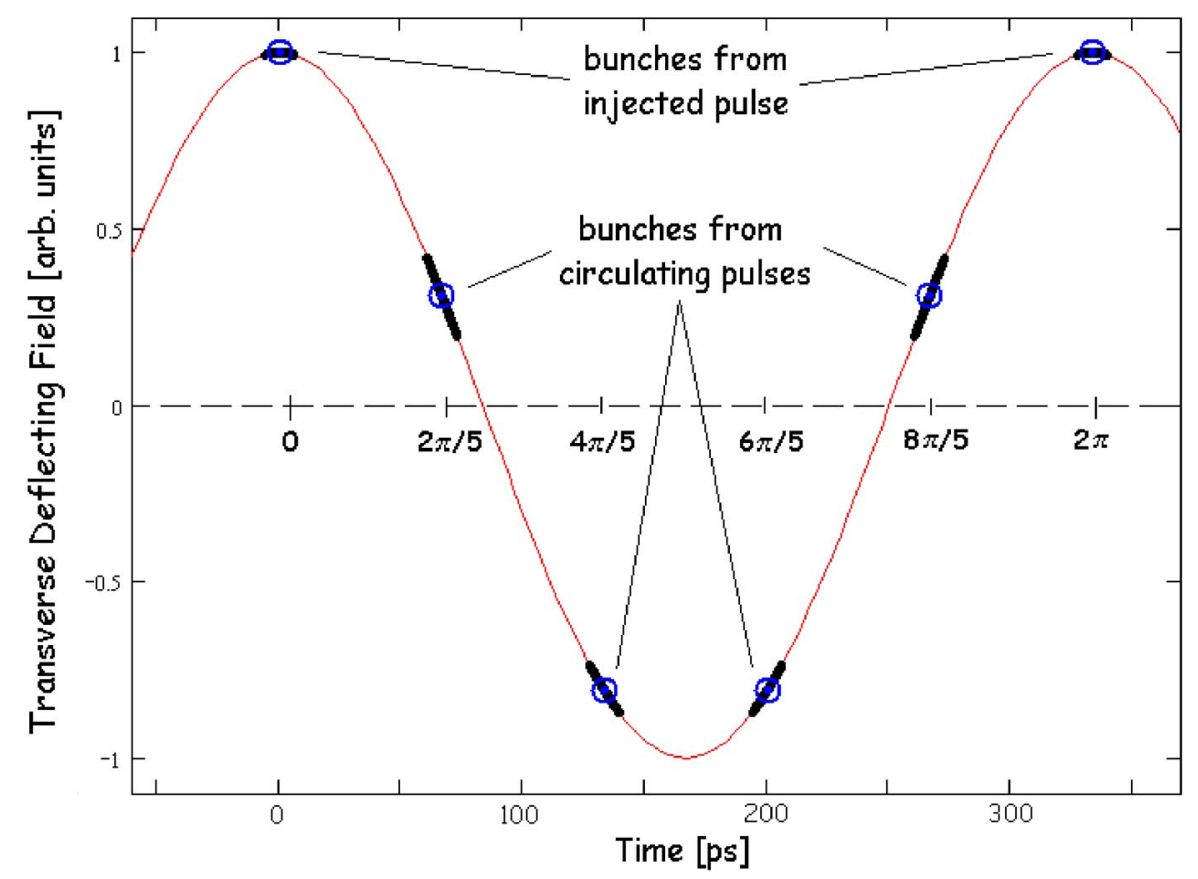

FIG. 4. (Color) Kick amplitude for injected and circulating bunches for a combination factor 5. The longitudinal extension corresponds to $\pm 2 \sigma$ for a bunch with a Gaussian distribution with $3 \mathrm{ps}$ rms bunch length. The circles indicate the centers of the bunches.

To limit the transverse extension, the bunches have to be kept short in length, and simulations have shown that about $6.5 \mathrm{ps} \mathrm{rms}$ is the maximum acceptable length for a combination factor of 5 .

\section{Beam optics and simulations}

The constraint on the bunch length has major implications on the beam optics in different parts of the CTF3 complex. In particular, this implies that both the injection line and the combiner ring have to be isochronous, which required several changes of the former LPI hardware. A complete description of the beam dynamics in the CTF3 Preliminary Phase can be found in Ref. [6].

\section{Linac}

The former LPI linac had been shortened. A matching section and a spectrometer line had been installed at the end of the linac. A redesign of the optics was carried out using the MAD program [7] without changing the existing quadrupole locations. Nevertheless, a few quadrupoles

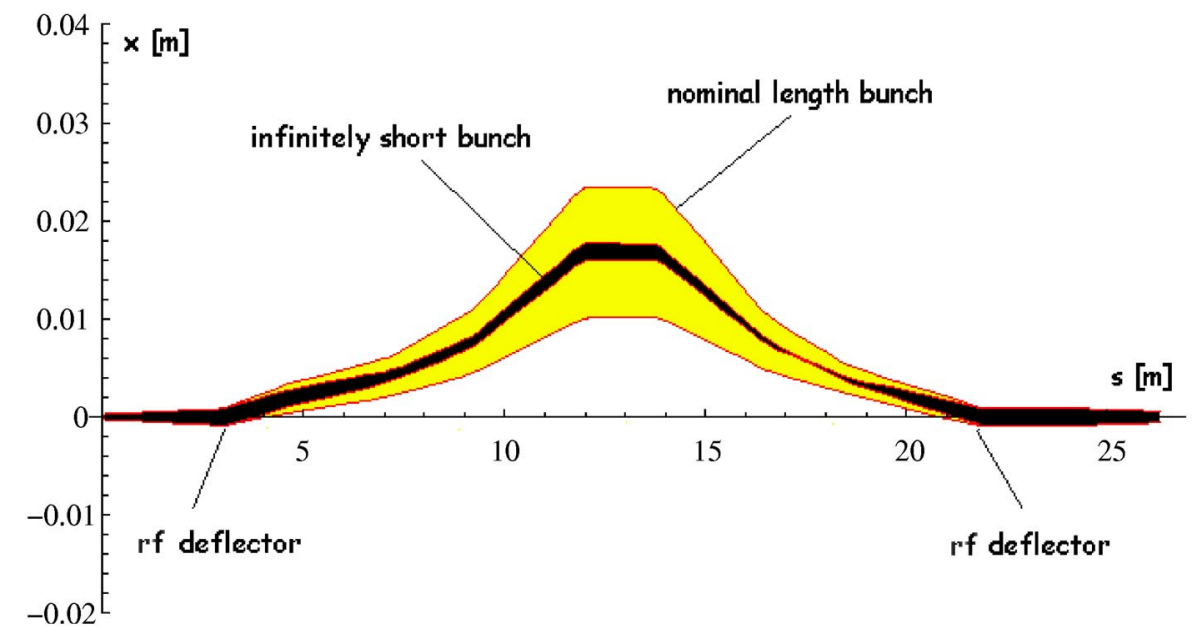

FIG. 5. (Color) Simulated beam envelopes of the bunches of Fig. 4 in the case of an infinitely short bunch and of a bunch length of $3 \mathrm{ps} \mathrm{rms}$. Both bunches have a normalized $\mathrm{rms}$ emittance of $15 \pi \mathrm{mm} \mathrm{mrad}$ and are located at $2 \pi / 5$ on the $\mathrm{rf}$ deflecting field. 


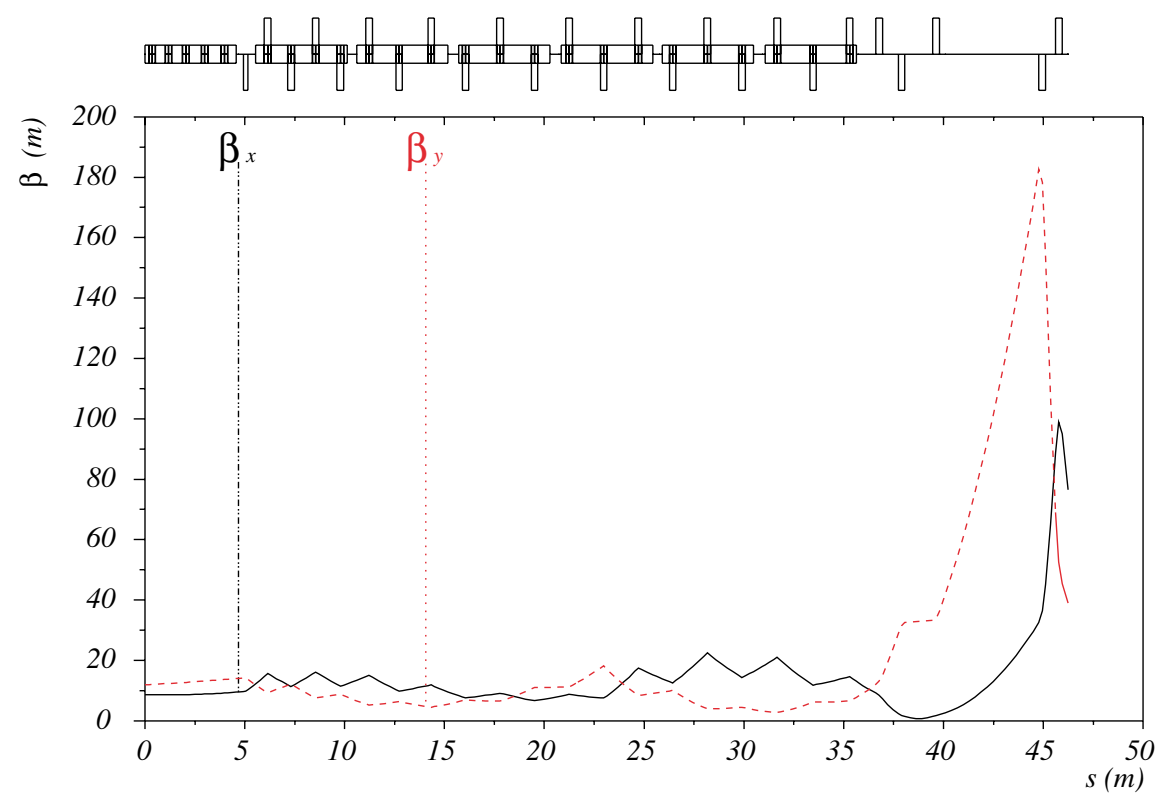

FIG. 6. (Color) Design optics of the linac and the matching section between the exit of the bunching system and the injection line. The horizontal (solid line) and vertical (dashed line) $\beta$ functions are shown.

were powered independently in order to provide flexibility. The matching section was made of five quadrupoles in order to adapt the Twiss parameters to the optics of the injection line. Figure 6 shows the optics along the linac.

\section{Transfer line}

The transfer line contained both horizontal and vertical bending magnets, allowing injection from the inside of the ring. It had to be achromatic to first order to fulfil the dispersion matching in both planes $\left(D_{x}=D_{y}=0\right.$ and $D_{x}^{\prime}=D_{y}^{\prime}=0$ ) at both ends. In this case the linear $6 \times 6$ transfer matrix $R$ has vanishing elements $R_{51}, R_{52}$, $R_{53}$, and $R_{54}$. As a result, the path length difference $c \Delta t$ of a given highly relativistic particle with a relative momentum offset $\Delta p / p$ with respect to the reference particle reduces to

$$
c \Delta t=R_{56} \Delta p / p
$$

This implies that $R_{56}=0$ to keep the bunches short for the combination process. Furthermore, the horizontal and vertical $\beta$ functions must be kept reasonably small, while allowing for matching at both ends. The solution found for the linear optics is shown in Fig. 7.

Even though first order isochronicity is very small, a second order effect remains. However, simulations have shown that this effect is small and does not lead to a significant bunch lengthening in the line.

\section{Combiner ring}

The optics of the combiner ring must also be isochronous to preserve the bunch length and spacing over up to five revolutions of the bunches for the combination. This means that the momentum compaction factor $\alpha_{c}$ must be zero. Simulations have shown that the condition $\left|\alpha_{c}\right| \leq$ $10^{-4}$ was sufficient in practice. In addition, the optics was designed to have $D_{x}=0$ in the injection region and small $\beta$ functions at the location of the rf deflectors in order to have a small beam size there. Some quadrupole families had to be decoupled and four quadrupoles were physically moved. One sextupole family was split in two to allow path length control up to second order for off-momentum particles. Tracking studies with this configuration have shown that the nonlinear effects are small and do not lead to any significant bunch lengthening. The corresponding optics is shown in Fig. 8.

In addition to the isochronous optics $\left(\alpha_{c}=0\right)$, another nonisochronous optics with $\alpha_{c}=0.034$ has been designed. This optics allowed the rf system to keep a stored beam circulating in the ring for diagnostics purposes, like closed orbit or tune measurements.

The injection using the $\mathrm{rf}$ deflectors had been modeled with MAD, where the rf deflector is described as a thin element with transfer matrix elements up to second order [8]. A particle distribution with realistic properties (transverse normalized $\mathrm{rms}$ emittance of $15 \pi \mathrm{mm} \mathrm{mrad}$, rms bunch length of $3 \mathrm{ps}$, relative energy spread of $0.2 \%$ rms) had been tracked to simulate the combination process for a combination factor 5 in the ring. Figure 9 shows the particle distribution after five turns at the extraction point.

The energy spread induced by the rf curvature in the linac leads to a variation of the deflection kick and results in an effective emittance blowup in the horizontal plane. This effect is illustrated in Fig. 10 which shows the phase 


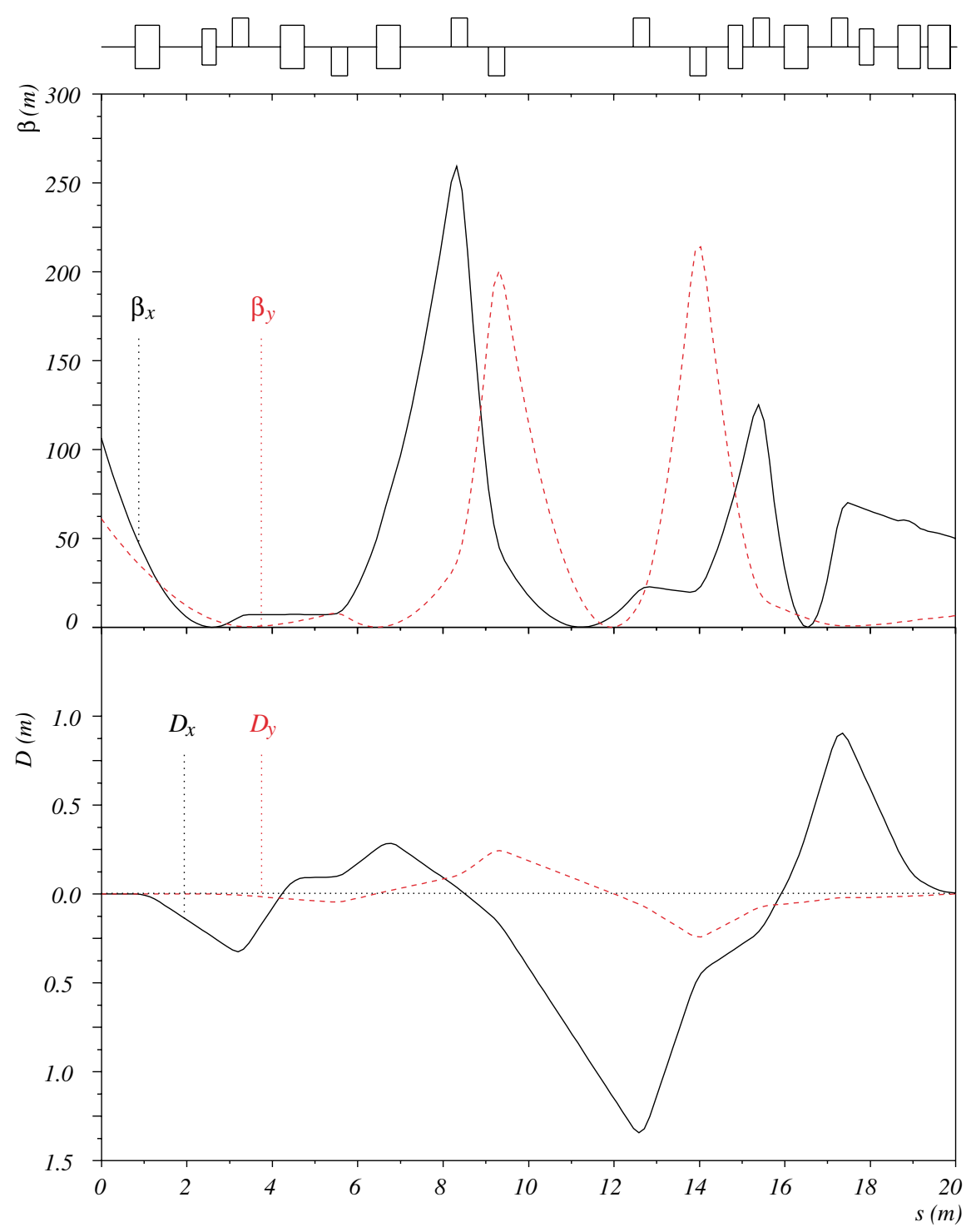

FIG. 7. (Color) Design optics of the injection line. The horizontal (solid line) and vertical (dashed line) $\beta$ functions are shown on the top graph, design dispersion functions for the horizontal (solid line) and vertical (dashed line) plane on the bottom.

space after five turns at the extraction point in comparison to an ideal injection.

Nevertheless, the statistical calculation of the emittance indicates a growth by $40 \%$, which is still acceptable for the demonstration of the bunch train combination.

\section{EXPERIMENTAL RESULTS}

\section{A. Commissioning with beam and measurements}

The linac and the combiner ring were commissioned in 2001 [9]. The rf deflectors were installed in the beginning of 2002. Several measurements were performed in order to prepare the bunch train combination [10]. The energy was measured after the bunching system and at the end of the linac. The beam optics was measured in the linac and found in good agreement with the design. The dispersion of the injection line was determined and optimized em- pirically to render the line isochronous. The betatron tunes of the ring were determined for different operating conditions and were in excellent agreement with the MAD machine model. The dispersion of the ring was measured for a nonisochronous optics and showed to be very close to the model.

\section{Beam energy measurements}

During the CTF3 operation, two series of energy measurements were performed, one at the exit of the bunching system and one at the end of the linac.

At the exit of the bunching system, the energy was measured by changing the current in two steering coils and by recording the data from a beam position monitor (BPM) downstream. This led to a measured momentum of about $6.5 \mathrm{MeV} / c$ at the buncher exit. 


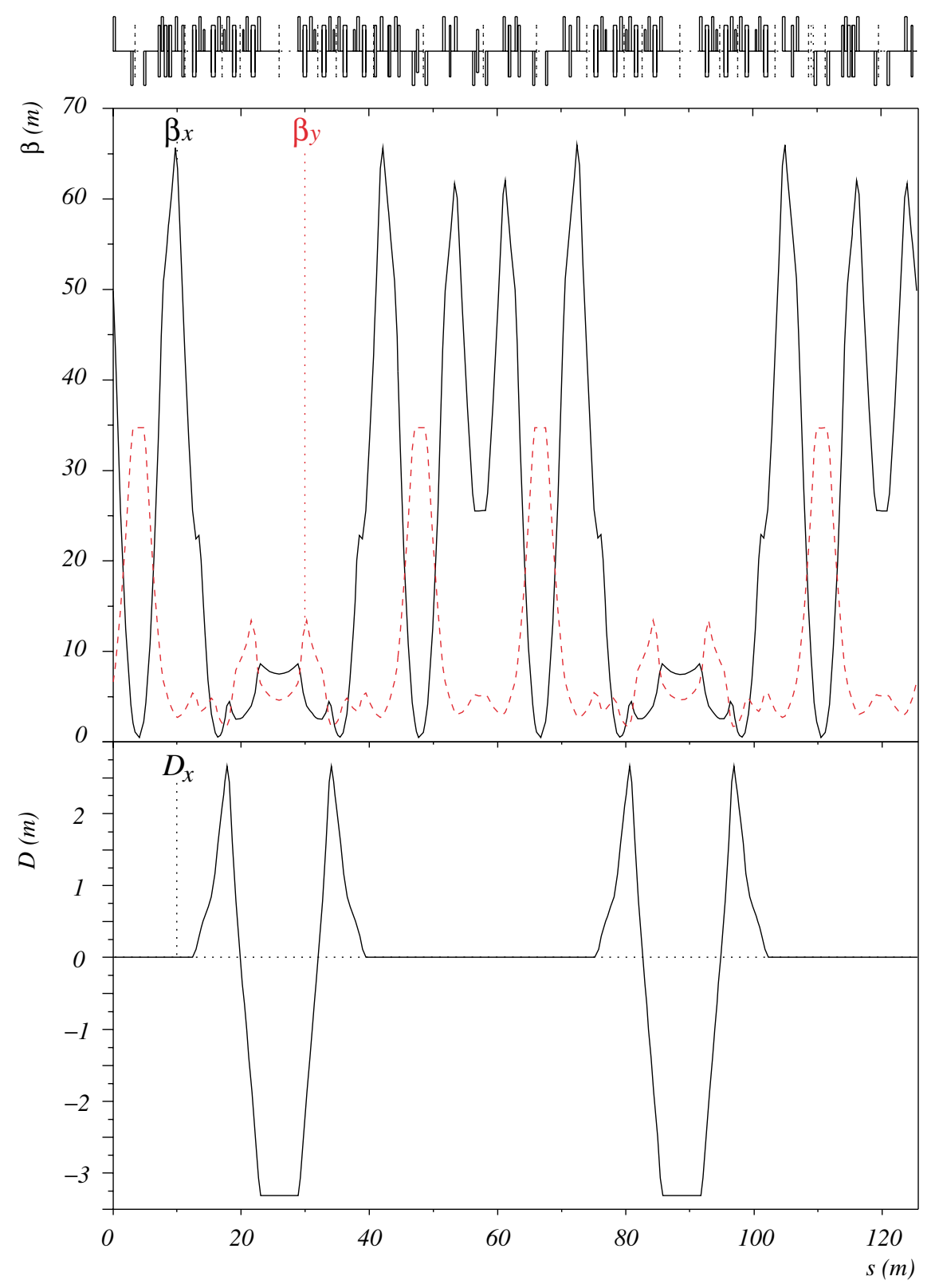

FIG. 8. (Color) Design optics of the isochronous combiner ring starting from the injection point. The top graph shows the horizontal (solid line) and vertical (dashed line) $\beta$ functions, the bottom graph the design horizontal dispersion function. The vertical dispersion is zero.

At the end of the linac, the beam energy was measured with different methods. It was derived from the measured rf power feeding the accelerating structures, it was directly measured in the spectrometer line in the matching section, and by using the injection line as a spectrometer. Some discrepancy between these three methods was found but not investigated in detail since the precise knowledge of the energy is not crucial for the bunch train combination. The linac was operated at an energy slightly lower than nominal as this turned out to be more stable. An energy of $332 \mathrm{MeV}$ (with about $1 \%$ uncertainty) was used during operation and for the subsequent calculations.

\section{Transverse beam dynamics}

Wire beam scanners (WBS) were widely used to measure the Twiss parameters in the linac. For this purpose, the current in a linac quadrupole was varied while observing beam transverse profile in a downstream WBS. The rms beam size was computed from the transverse profiles, and the Twiss parameters were derived from the data.

In general, scans performed during the operation in 2002 [10] were very similar to the ones obtained in December 2001 [11], which demonstrated the reproducibility of the machine. 


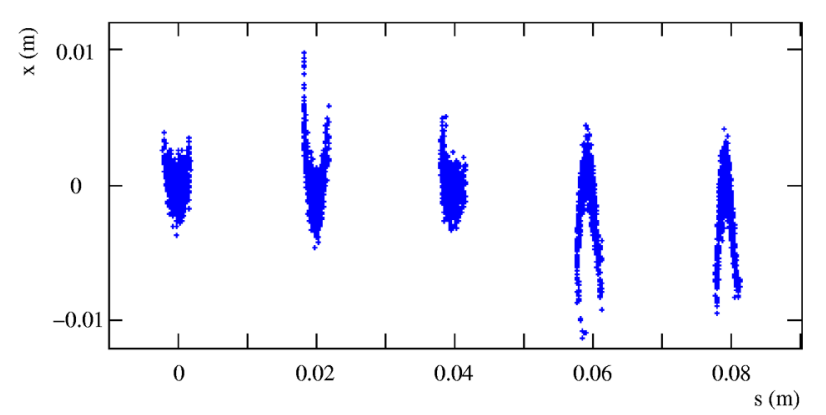

FIG. 9. (Color) Horizontal transverse versus longitudinal position for a combination factor 5 in the combiner ring with realistic energy spread for the injected bunches.

Two sets of quadrupole scans were performed at different locations in the linac. Using the MAD model of the linac, the measured Twiss parameters of the downstream scan were backpropagated to the quadrupole used for the first set of scans. The data fit well the results of the upstream scans (see Fig. 11), showing the validity of the MAD model that includes the acceleration.

The optics of the matching section was adapted according to the measured Twiss parameters in order to obtain the nominal Twiss values at the entrance of the injection line.

\section{Dispersion measurements in the injection line}

Dispersion measurements were performed in the injection line, in order to verify that the optics model corresponds to the measured dispersion pattern. For this measurement, three scintillator screens with their associated cameras, as well as two-beam position monitors, were used. The currents in all magnetic elements were changed in order to simulate relative energy variations between $-2.7 \%$ and $+2.7 \%$, while the corresponding beam positions were recorded in each diagnostic tool. Figure 12 shows a comparison between the dispersion curves given by the MAD model and the measured values.

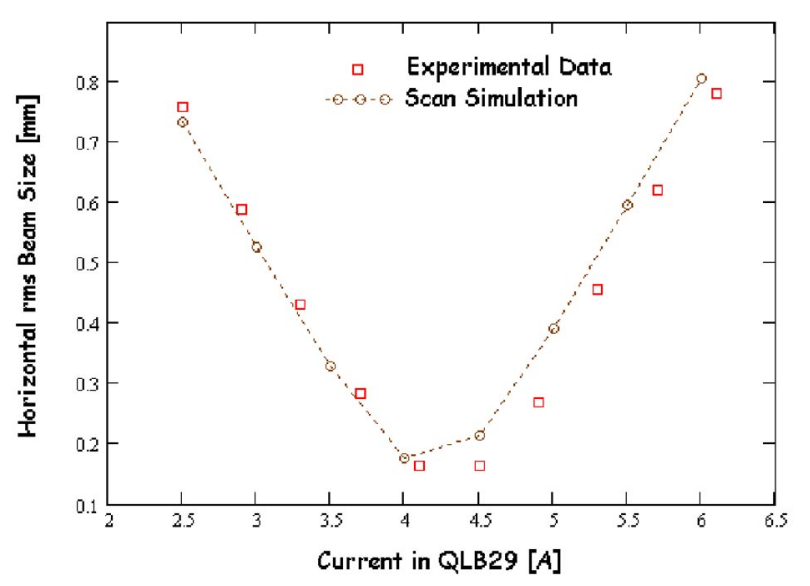

FIG. 11. (Color) Transverse beam size measured by a wire beam scanner as a function of the quadrupole current. The measured data points (squares) are compared to a simulated scan (circles) that is based on the initial Twiss parameters backpropagated from a downstream measurement.

In the horizontal plane, there is very good agreement between the measured and the simulated values of the dispersion at each measurement point.

In the vertical plane, the agreement is also good, except for a discrepancy between the measured and expected values of the dispersion in the last scintillator screen. The measured values in both planes could be reproduced in the model by changing the gradient of one quadrupole, indicating a possible current calibration error.

\section{Measurements in the ring}

Tune measurements in the combiner ring using two different methods were compared in various conditions. The first method uses the former standard LPI measurement system, which consisted of a pickup electrode combined with a spectrum analyzer. The other method is based on a Fourier transform analysis (performed with a digital oscilloscope) of the horizontal and vertical signals of beam position monitors in the ring at injection.
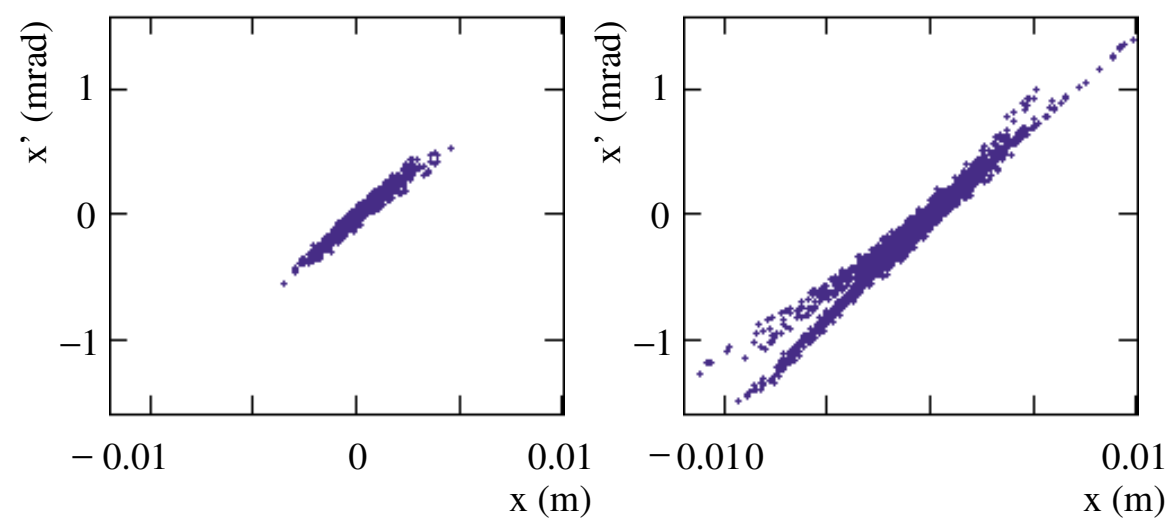

FIG. 10. (Color) Horizontal phase space at the extraction point after five turns. The left plot corresponds to the case of a perfectly uniform kick at injection; the right plot is obtained when using rf deflectors for a bunch combination factor of 5 . 


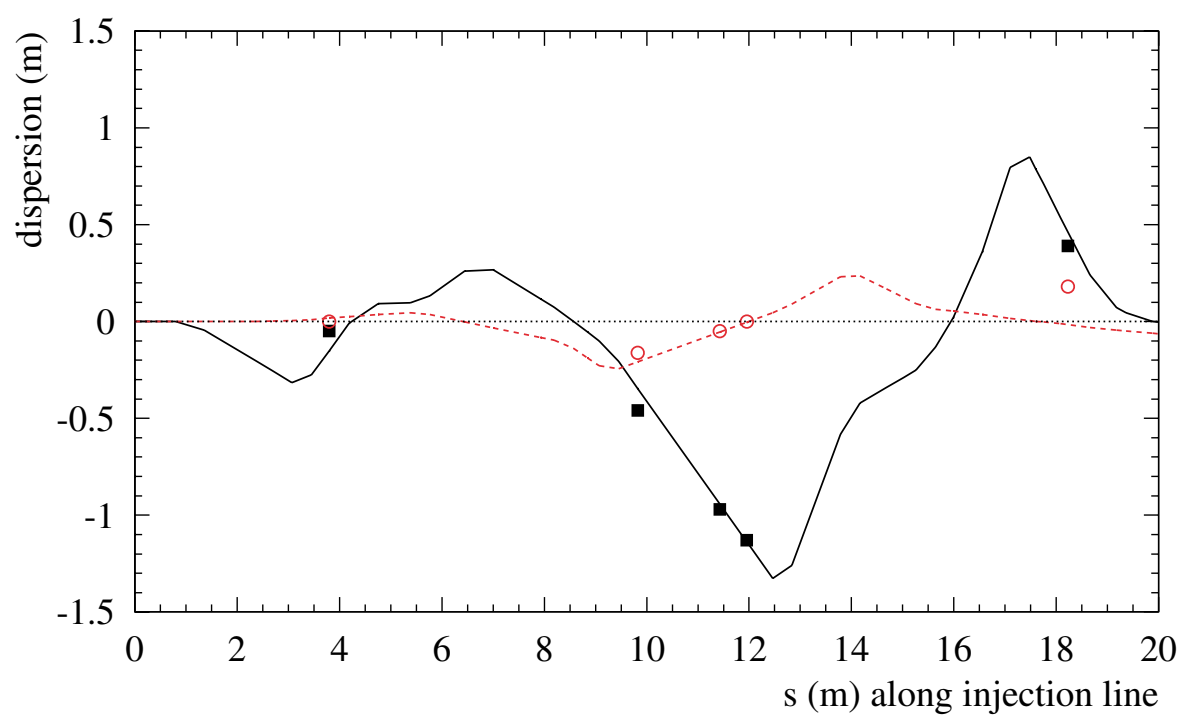

FIG. 12. (Color) Dispersion measurements in the injection line. The full squares are the experimental points in the horizontal plane and the solid curve is given by the model for the corresponding current settings. The open circles and the dashed line are measurements and expectations from the MAD model in the vertical plane, respectively.

The Fourier transform analysis has the advantage that it does not require a stored beam in order to perform the tune measurement: it could thus be used with the isochronous configuration as well, contrary to the spectrum analyzer system that could only be used with a stored beam due to its long integration time.

These two methods and the MAD model were crosschecked with a stored beam in the nonisochronous accumulation mode. The current settings of several quadrupole families were varied and the measured tune was compared to the model (see the example in Fig. 13).
The agreement between the two measurement methods was very good, typically of the order of 0.01 which coincides with the resolution of the Fourier method. Therefore, we validated this method and used it for further measurements with the isochronous optics.

The MAD model also agreed very well with the measurements. For both the accumulation and the isochronous modes, the differences between the expected and measured tune values were typically 0.01 to 0.02 , comparable to the precision of the measurement.

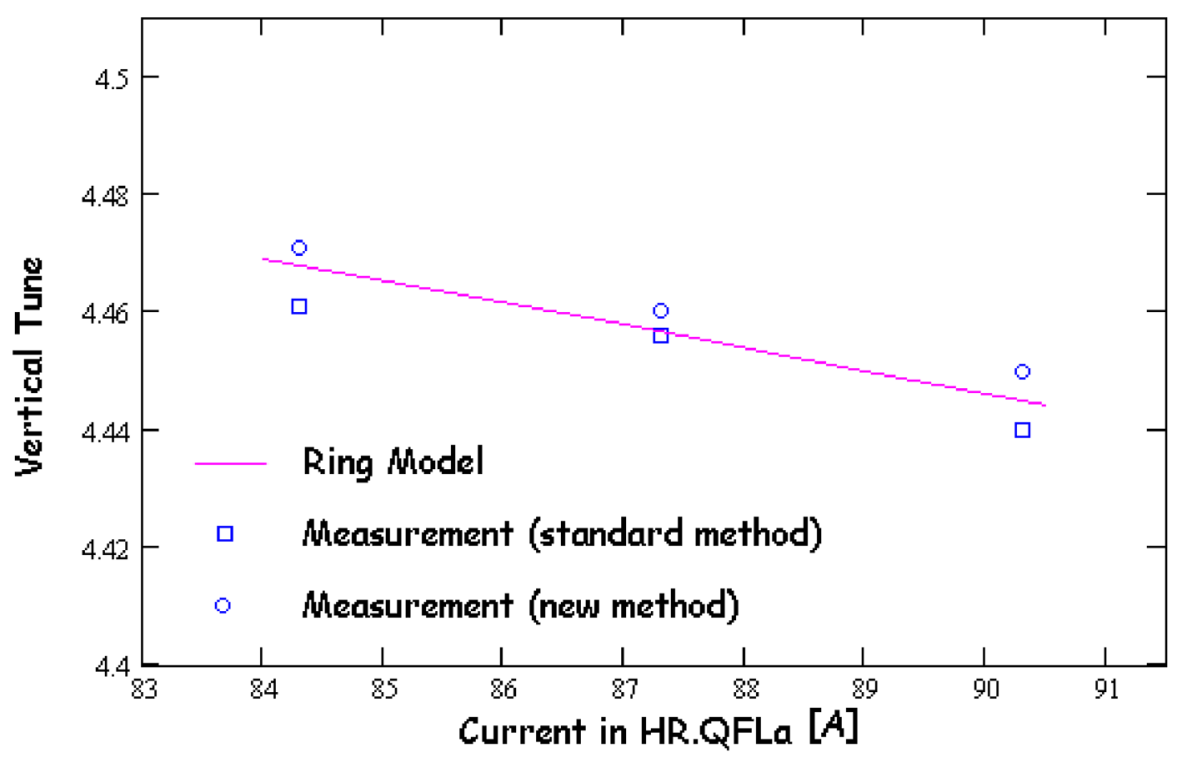

FIG. 13. (Color) Vertical tune measurements in the ring in accumulation mode as a function of the current in one quadrupole family and comparison between two methods and the MAD model. For clarity, the error bars on the measurement points $( \pm 0.01)$ are omitted. 


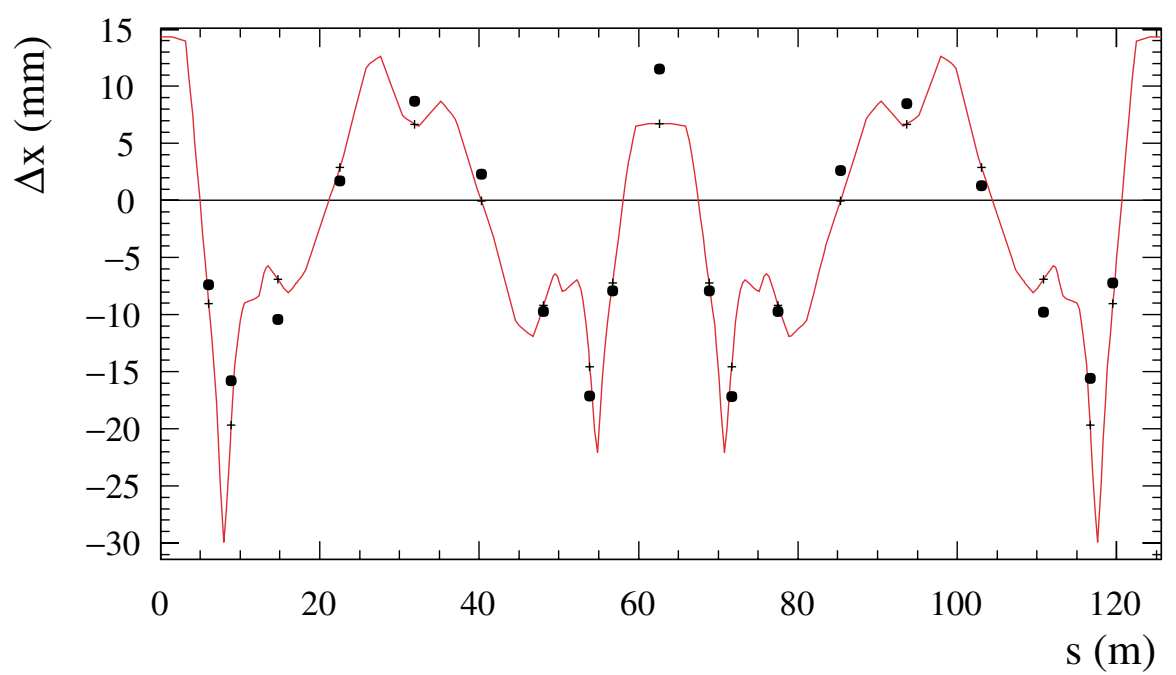

FIG. 14. (Color) Measured horizontal closed orbit difference (black points) for a change in frequency from 19.089942 to 19.083 229 MHz. The solid line corresponds to the expected closed orbit difference, proportional to the dispersion calculated from a model with the measured magnet currents.

For a further validation of the ring optics, closed orbit measurements were performed in the accumulation mode at various frequencies of the rf cavity in the ring, in order to determine the dispersion function. Figure 14 shows an example of the measured horizontal closed orbit difference, proportional to the dispersion, for a change in frequency from 19.089942 to $19.083229 \mathrm{MHz}$.

The agreement with the curve calculated with MAD using the experimental quadrupole currents is quite good. Consequently, we believe that the model gave a good estimate of the momentum compaction factor $\alpha_{c}$ which needs to be smaller than $10^{-4}$ for the isochronous operation.

\section{Bunch length and isochronicity measurements}

As mentioned before, the bunch length is a critical issue for the combination process. The bunches must be kept short in order to limit the variation of the injection kick strength and the transverse extension in the injection region. With the rf deflector injection scheme, simulations have shown that $6.5 \mathrm{ps} \mathrm{rms}$ is the maximum acceptable bunch length for a combination factor 5 .

Experimentally, the bunch length was first measured at the end of the linac, using a transition radiation screen in conjunction with a streak camera. Taking into account the measurement resolution, a value of the order of $3 \mathrm{ps} r \mathrm{rs}$ was found.

Another method to measure the bunch length at the end of the linac with a higher precision was used to confirm the streak camera measurements. It is based on the relationship between the energy spread and the phase of the accelerating rf wave. Provided the charge is low enough, beam loading is negligible, and the energy spread for a given bunch length depends on the position of the bunch on the rf cosine wave. During the measurement, the phase between the buncher and the accelerating structures is varied, while the energy spectra are monitored downstream in a spectrometer. Assuming a Gaussian longitudinal profile for the bunches, the expected energy spread can be calculated and compared to the experimental values in order to assess the bunch length. A constant contribution was added in quadrature to the calculated energy spread to take into account several effects influencing the resolution ( $\beta$ function, uncorrelated energy spread from the buncher, beam jitter). The results are presented in Fig. 15 which shows that the measured energy spread is compatible with the simulations for a bunch length of $2.0 \mathrm{ps} \mathrm{rms}$ with a precision of \pm 0.4 ps rms.

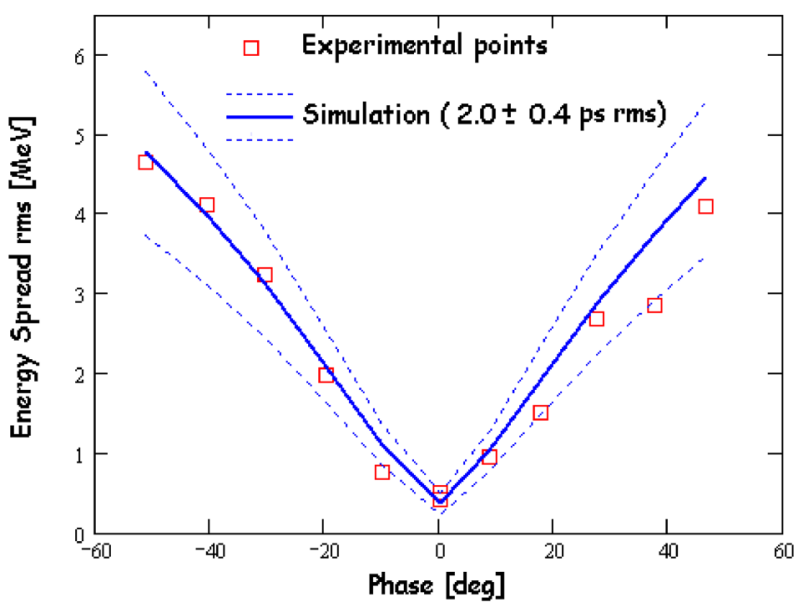

FIG. 15. (Color) Energy spread as a function of the rf phase shift for the accelerating structures and simulations for a bunch length of $(2.0 \pm 0.4) \mathrm{ps}$ rms. 

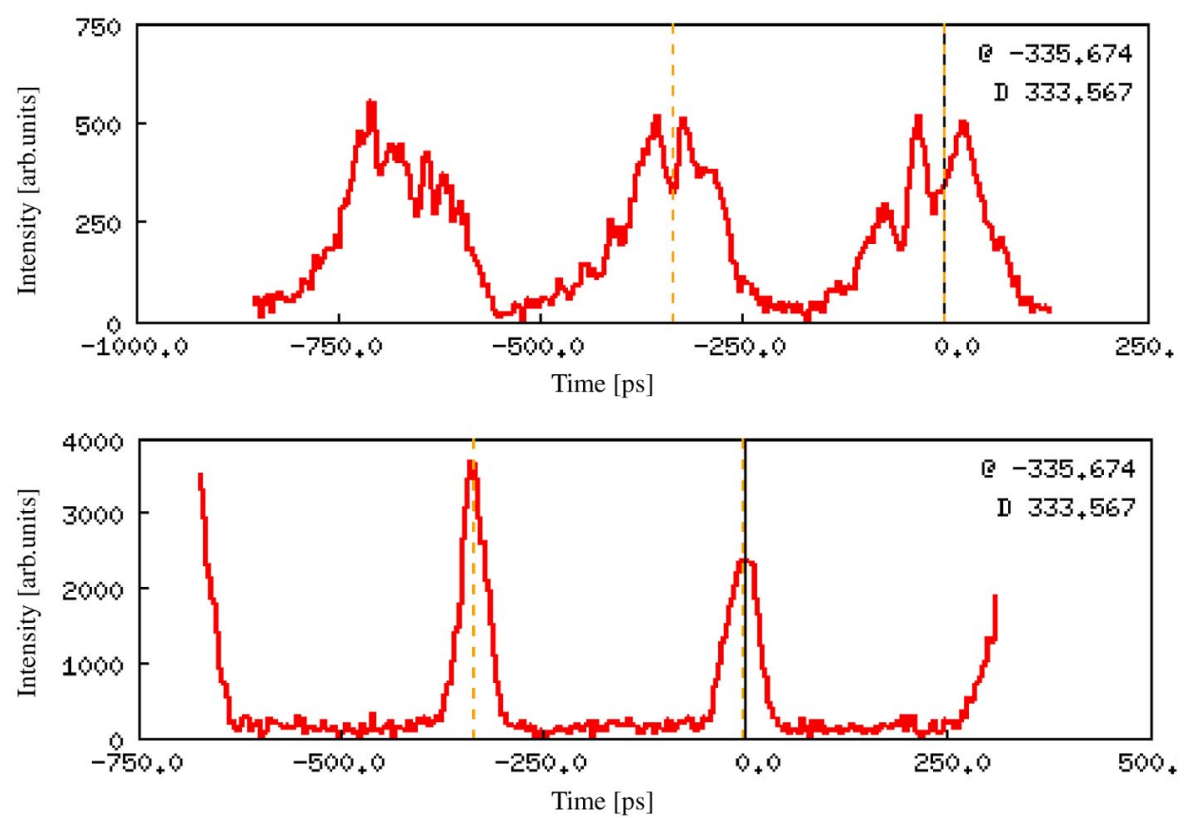

FIG. 16. (Color) Longitudinal intensity profile as measured with the streak camera. The top profile is taken during the second turn for the nonisochronous optics. The bottom image shows the distribution in the 60th turn for the isochronous optics.

The same measurement was repeated with different charges per bunch (ranging from 0.075 to $0.1 \mathrm{nC}$ ) and gave similar results, i.e., a bunch length of 2 to 3 ps rms. The measured values of the bunch length and energy spread were compatible with those obtained with the streak camera measurements in the linac and also with previous measurements made in the LPI complex, where the same bunching system was used $[12,13]$. The upper limit for the bunch length at the end of the linac is therefore around 3 ps rms, well below the limit of $6.5 \mathrm{ps}$ rms.

The results of these measurements were used in the simulations presented in Sec. IIC and allowed us to determine the level of isochronicity required in the injection line and in the ring.

There was no possibility to measure the bunch length directly in the injection line. So the isochronicity had to be estimated indirectly from dispersion measurements in the line or bunch length measurements in the ring. The isochronicity in the ring could be carefully optimized by observing the time structure of the synchrotron light emitted in a bending magnet of the ring with the streak camera. The longitudinal bunch profile broadens quickly for a nonisochronous optics due to the energy spread within the bunch. Figure 16 shows an example of a profile taken at the second turn with the nonisochronous optics compared to a profile taken after 60 turns for a welloptimized isochronous optics.

As shown in Fig. 17, the transition from a positive to a negative momentum compaction factor in the isochronous optics is clearly visible on the streak camera images when changing the current in one quadrupole family. Indeed,
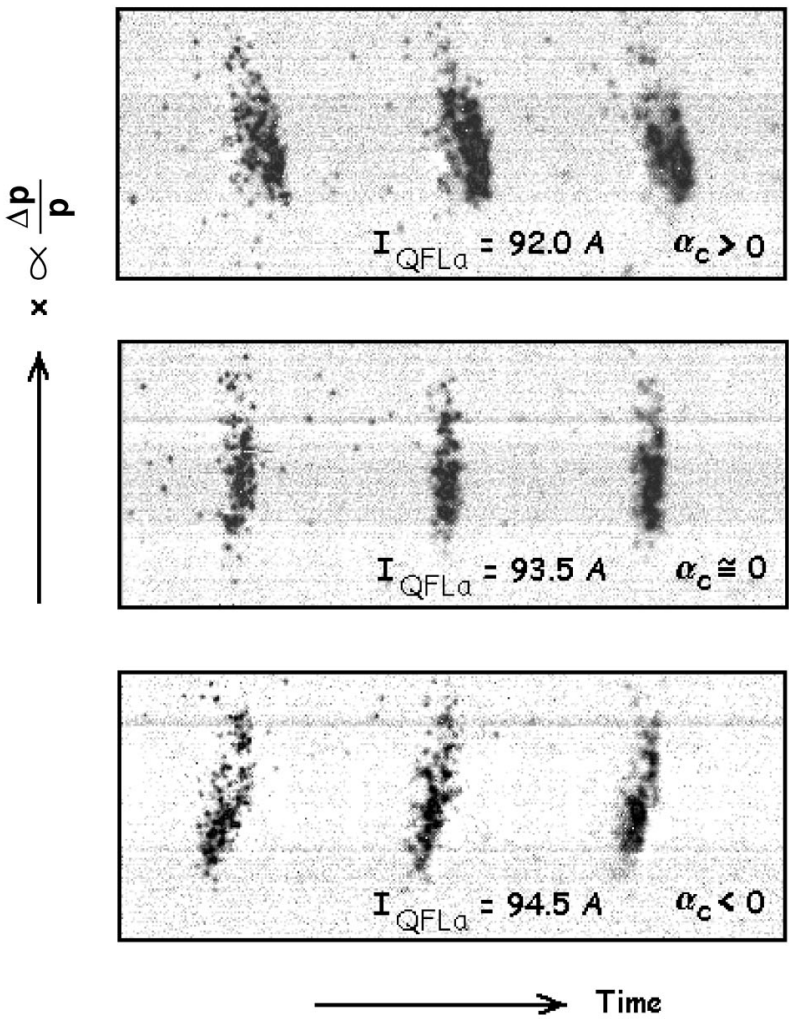

FIG. 17. Transition from positive to negative momentum compaction factor $\alpha_{c}$ seen on streak camera images for different settings of one quadrupole family. The images are taken during the tenth turn at a location with nonzero dispersion. The horizontal position $x$ is dependent on the energy, so the timemomentum correlation becomes apparent. 
the nonzero momentum compaction factor introduces a time-momentum correlation in the bunches which depends on the sign of $\alpha_{c}$.

After careful optimization, the bunch length measured on the streak camera profiles was of the order of $4 \mathrm{ps} \mathrm{rms,}$ not changing over several turns and comparable with the one obtained at the end of the linac. Thus, within the resolution limit of the streak camera, no significant bunch lengthening was observed, neither in the injection line nor in the ring, proving that the isochronicity was obtained at the required level for the bunch train combination.

\section{B. Bunch train combination results}

A first demonstration of the bunch train combination process, for a combination factor $N=4$, was obtained in June 2002. However, the performances were still limited by losses in the rf deflectors. The ring transverse acceptance was indeed not sufficient to avoid losses at injection, even for the standard injection procedure, and the setting up of the combination process was difficult. In August 2002, the CERN deflectors were replaced with new ones, built by INFN-Frascati, which have an almost twice larger aperture. The increased transverse acceptance and a better transverse beam matching allowed the losses to be reduced right away and it became possible to change the beam orbit in the first turns after injection over a large range. This in turn allowed for the development of a reliable setting-up procedure for the bunch train combination (described in the following) and the losses were rapidly brought to zero. The bunch train combination for $N=5$ was then set up, and again in this case the efficiency was almost $100 \%$. A combination factor 3 was also obtained, but was neither optimized nor studied in detail. During the last operation period, the combination performances were studied in more detail, with particular attention to the critical issues of bunch phase and transverse position stability.

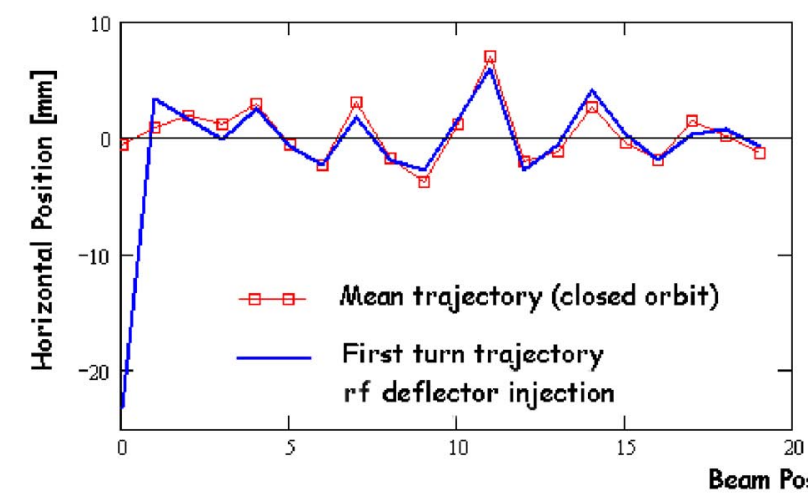

FIG. 18. (Color) Reference closed orbit and first turn trajectory in the isochronous ring in both planes. The reading in the first BPM shows the beam position at injection, before the deflector kick.

\section{Injection with an rf deflector}

The first step in the bunch train combination process was to demonstrate the injection process with one rf deflector. This process is analogous to a single-turn injection with a fast kicker. In this scheme, the beam is deflected by the injection septum towards the closed orbit, which is reached at the kicker location. The kicker then compensates the residual kick (of the order of $4 \mathrm{mrad}$ in our case) so that the beam follows the closed orbit. When the kick is provided by an rf deflector, it changes rapidly in time, but all bunches in a train, having the same repetition frequency as the $\mathrm{rf}$ in the deflector, arrive with the same phase and experience the same kick.

In CTF3, one of the two deflectors (called first deflector or injection deflector in the following) was installed in the ring injection straight section, after the septum and close to the existing fast kicker. The latter was kept in place, in order to allow for standard single-turn injection.

A single train was produced by the gun and accelerated in the linac. It was injected into the ring using the standard single-turn injection scheme. The fast kicker was then switched off, and the injection deflector was powered. Since the filling time of the $\mathrm{rf}$ deflectors is smaller than the revolution period (about 50 ns with respect to $420 \mathrm{~ns}$ ), a proper choice of the rf pulse length and timing results in a kick at the first passage only. The rf phase was then tuned in order to obtain the maximum deflection (i.e., the bunches arrive at the crest of the $\mathrm{rf}$ wave). The kick amplitude and the injection angle provided by the septum could then be adjusted. The latter indeed had to be modified in order to compensate for the small difference in the betatron phase advance between the injection deflector and the fast kicker.

After optimization, the injection performances were the same as for the standard single-turn injection. In particular, the beam could be kept circulating in the ring for more than 100 turns with no measurable losses and the residual oscillations around the closed orbit were close to the limit of the beam position monitor resolution (see Fig. 18). It should be noted here that the closed orbit

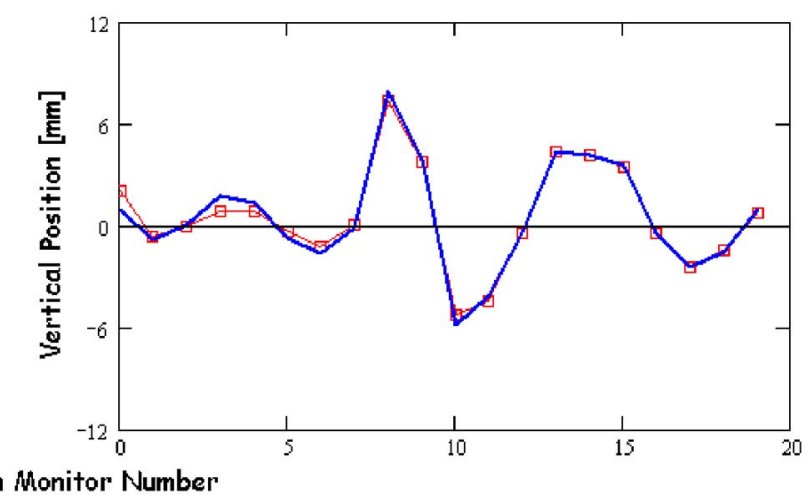


itself showed large oscillations. Because of a limited number of orbit correctors (four horizontal, two vertical), the orbit could not be well corrected.

In standard operating conditions, the power required to inject the beam was about $7.2 \mathrm{MW}$ at the structure input. This value is in very good agreement with our expectations.

\section{Bunch train combination setting-up procedure}

The bunch train combination process is controlled by five parameters: the amplitudes and phases of the rf field in each of the two rf deflectors and the common $\mathrm{rf}$ frequency for the linac and the deflectors. In order to close the time-dependent bump, the rf field must have the same amplitude in both deflectors and the same phase when the beam passes. Since the path length in the isochronous ring is constant, the rf frequency must have a precise value for a given combination factor, as expressed in Eq. (1).

A procedure was developed in order to optimize the five parameters. This procedure minimizes the injection error with respect to the closed orbit of the ring and is described in detail in Ref. [14].

The following procedure describes the bunch train combination for a multiplication factor of 4 . In this case, it is simplified because the beam arrives after one turn at the zero crossing of the deflecting field in the second rf deflector and it is therefore not deflected. This procedure is based on the use of only one bunch train in the linac. Once completed, the four trains are sent in the machine for the combination.

Initially, one deflector only is used in order to inject the beam on the ring closed orbit. To do so, the rf pulse length and timing are adjusted such that there is no rf power in the second deflector when the beam, after one turn, passes through it.

The five parameters that control the bunch train combination are adjusted as follows:

The phase in the first deflector is varied while recording the horizontal beam position in a BPM located downstream of the deflecting structure. The horizontal position of the beam describes a cosine curve corresponding to the rf field in the structure, and the phase corresponding to the crest can easily be identified.

The kick amplitude in the first deflector is varied until the beam passes in the ring beam position monitors at the same positions as for the closed orbit. The closed orbit is calculated from an average over ten successive turns to average out betatron oscillations. Figure 18 shows the mean trajectory which defines the closed orbit and the trajectory at the first turn when using the rf deflector for injection.

The $r f$ frequency is adjusted by looking at the second turn in the ring with only the first deflector in operation (i.e., with a maximum attenuation in the rf-network branch feeding the second deflector). After one turn, for a combination factor 4 , the bunches must arrive in the deflector at the zero crossing of the rf field, so that they are not deflected. The frequency of the whole complex is therefore tuned so that the second turn trajectory is the same as for the first turn.

In the case of a multiplication factor of 4 , after one turn in the ring, the injected bunches must also arrive in the second $\mathrm{rf}$ deflector at the zero crossing of the rf field. The attenuation is thus set to its minimum value so that the second $\mathrm{rf}$ deflecting structure is fed by the maximum available power. The phase in the second deflector is then varied until the beam does not experience any deflection at the second turn and therefore follows the closed orbit. A difference in the kick amplitudes of the two deflectors is not important at this stage. On the contrary, having the maximum available power in the second deflector enhances the sensitivity to the phase.

After two turns in the ring, the bunches arrive in the deflectors at the crest of the rf wave, with the opposite kick compared to the injection. At this point, they are deflected by the second deflector away from the septum and the injection deflector must close the bump. The amplitude in the second deflector is varied until the beam follows the closed orbit in the ring (outside the injection region) during the third turn, after the bump.

It must be noted that one or two iterations of the procedure were often necessary to achieve the best optimization since, for instance, tuning the frequency of the complex can induce small changes in the rf phase of the klystron, which then needs to be optimized again. Once this procedure is completed, it is possible to inject four pulses in the ring, as required for the combination.

\section{Bunch train combination efficiency}

The combination factor 4 was obtained with a frequency $f_{4}=2.998585 \mathrm{GHz}$. After the optimization, the bunch train combination showed a $100 \%$ efficiency. The charge multiplication could be observed on the intensity signal of beam position monitors in the ring. Figure 19 shows that the charge increases each time a new train of bunches is combined with those already circulating in the ring. The increase is not exactly linear due to pulse-topulse variations of the beam current delivered by the thermionic gun.

The evolution of the time structure of the electron pulse was observed with the streak camera. Figures 20 and 21 show typical images and their corresponding intensity profiles. Also in this case, the bunch-to-bunch variations shown in the intensity profiles are due to variations in the charge and the longitudinal profile of the pulses, already present in the linac.

For a combination factor 5 , the frequency was changed according to Eq. (1) and further optimized experimentally to $f_{5}=2.998715 \mathrm{GHz}$. Again, a $100 \%$ combination 


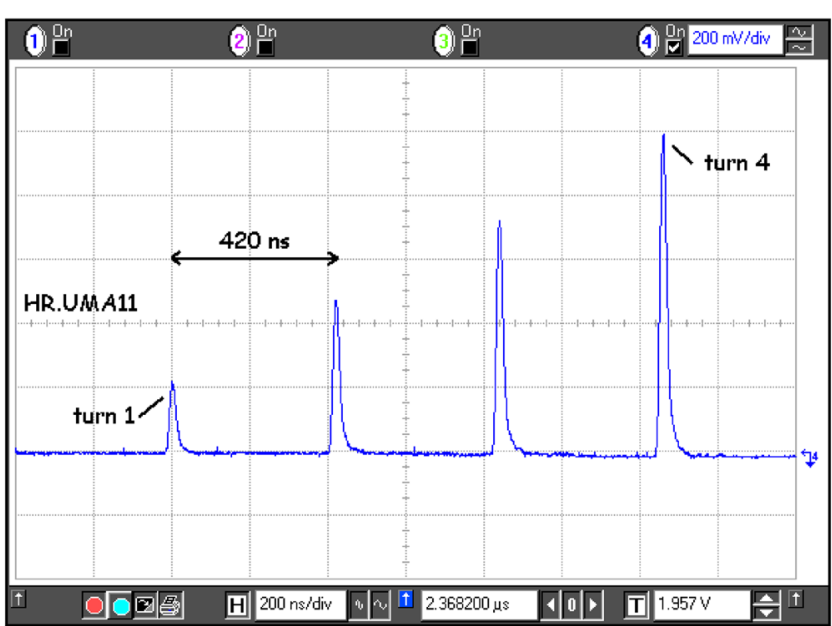

FIG. 19. (Color) Intensity signal of a beam position monitor in the ring for a bunch combination with a factor 4 .

efficiency was obtained. Figure 22 shows an example of an intensity profile of the combined bunch trains.

\section{Bunch train combination performances}

The bunch train combination performance was further studied in order to assess the limitations of the process for high-frequency $\mathrm{rf}$ power production in the following phases of the CTF3 project. The most relevant parameter for rf power production is the bunch length. Within the resolution limit of the streak camera, no bunch lengthening was observed (see Sec. IIIA5). In order to obtain a good flattop in the rf pulse produced by a drive beam, the current along the combined pulse must be constant. As discussed previously, this was not the case in the CTF3 Preliminary Phase, but the origin of bunch-to-bunch charge variations was tracked back to the gun current and timing jitter. In the following phases of CTF3, a new gun will be used, with longer pulses and designed for a much more stable current.

Other important effects that can spoil the power production efficiency are the following:

Bunch-to-bunch variations in transverse position.The aim of the combination process is to put the pulses onto the same orbit. Variations in transverse position are equivalent to an effective emittance growth. They can complicate the transport of the beam after extraction from the ring and give rise to transverse instabilities.

Bunch-to-bunch distance variations.-The distance between consecutive bunches after combination must be constant and equal to the rf wavelength to be produced (or an integer multiple). Variations will cause a loss of efficiency.

These variations were studied with the streak camera analyzing the synchrotron radiation emitted in the ring.

Bunch-to-bunch variations in transverse position were observed in the streak camera images, especially before
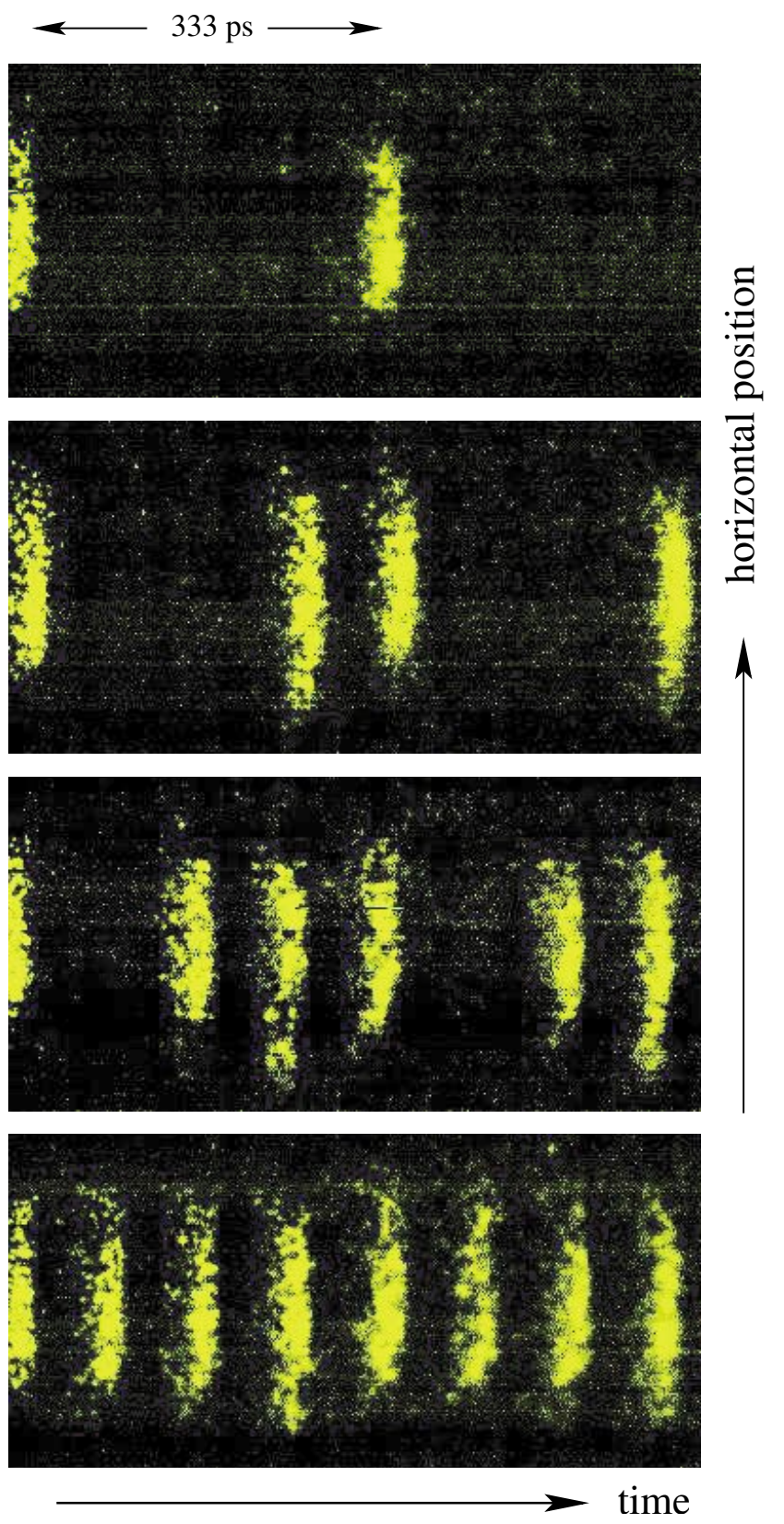

FIG. 20. (Color) Bunch train combination with a factor 4, as observed with a streak camera. The horizontal axis represents time; the vertical axis corresponds to the horizontal position. The images from top to bottom are taken over four consecutive turns.

having established a reliable setting-up procedure for the combination process. In all cases, bunches belonging to the same train have strongly correlated positions. This is visible, e.g., in Fig. 23, which shows a streak camera image of the beam after combination (factor 5), observed at a location where the dispersion function is maximum $\left(\left|D_{x}\right| \simeq 3 \mathrm{~m}\right)$.

The presence of train-to-train energy variations, caused by phase and amplitude fluctuations along the rf 

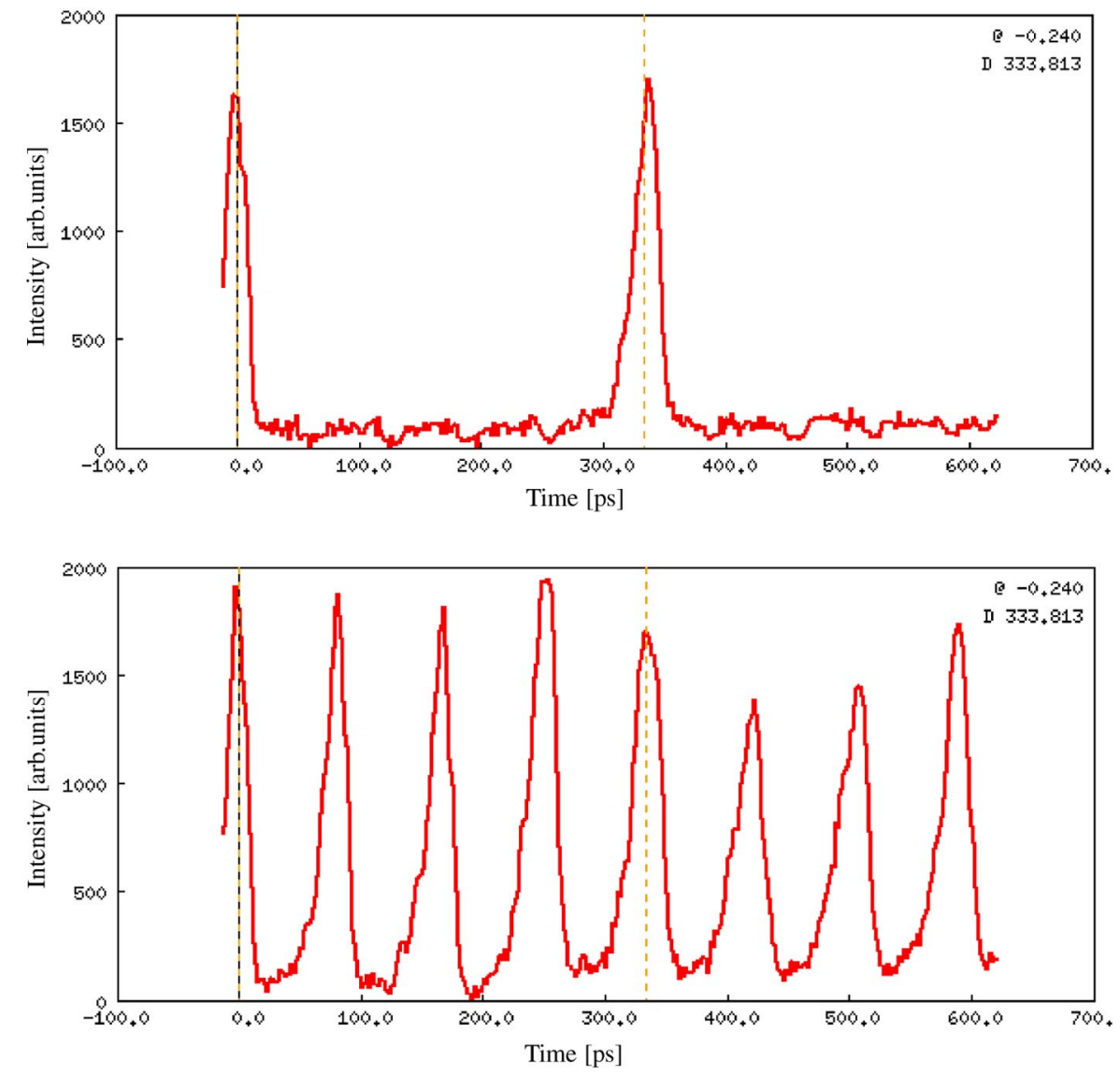

FIG. 21. (Color) Longitudinal intensity profiles for a multiplication factor 4 . The two images correspond to the injection of one and four bunch trains, respectively. Amplitude variations are due to bunch current variations already present in the linac.

pulse, was identified as the main cause since the largest oscillations were observed at the observation point with the largest dispersion. The energy difference gives rise to a position difference because of the nonzero dispersion. For large dispersion, the horizontal beam size is dominated by the energy spread, and it can be seen from Fig. 23 that the train-to-train energy difference is smaller than the single bunch energy spread. This kind of variation vanishes in regions with zero dispersion and does not correspond to an increase in transverse emittance.

Another source of fluctuations was injection errors, in position or in angle. The injected bunches oscillate around

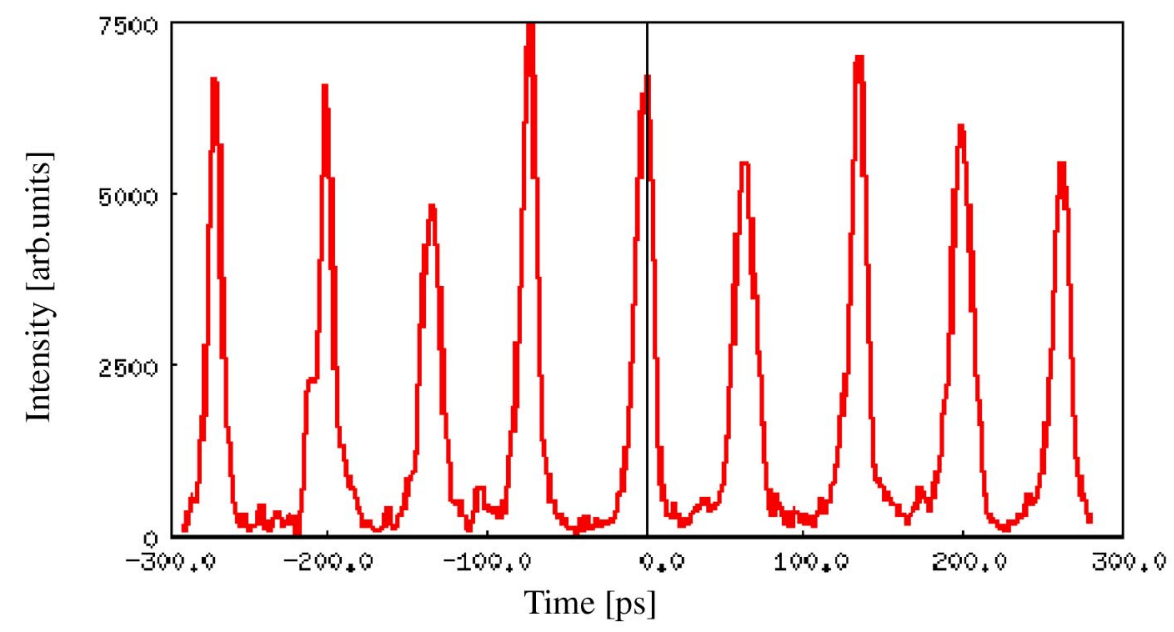

FIG. 22. (Color) Longitudinal intensity profiles for a multiplication factor 5 . 


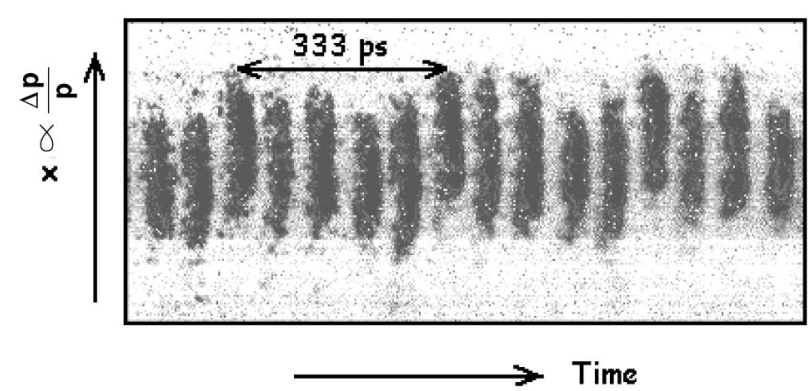

FIG. 23. Streak camera image of a combined beam (factor 5), showing bunch-to-bunch variations in transverse position. The dispersion function at the observation point is $\left|D_{x}\right| \simeq 3 \mathrm{~m}$. A periodicity corresponding to the combination factor is clearly visible.

the closed orbit and their position at the observation point then varies from turn to turn. Since different trains reach the observation point after a different number of turns, their bunch position appears to be different on the streak camera image. This phenomenon can be observed even at a point in the machine where $D_{x}=0$ and is indeed equivalent to a growth of the overall transverse emittance. However, these oscillations were strongly reduced when the setting-up procedure was systematically applied, which implies a minimization of turn-to-turn orbit differences. The residual oscillations were then typically only a fraction of the rms beam size.

Initially, the main concern was caused by the observed variations in bunch spacing. Figure 24 shows a streak camera image of a bunch combination with a factor 5 observed during the fifth turn. The bunch spacing, which should be $67 \mathrm{ps}$, is clearly not constant at that point. This image was taken before the injection optimization; also in this case the effect was significantly reduced afterwards.

Since the precision of the bunch spacing after the bunch combination is a crucial issue for the CLIC drive beam scheme, a specific experiment was carried out to measure the bunch spacing variations and compare them with the expectations from the model of the ring. Two bunch trains were injected and combined in the ring, and the bunch spacing was observed turn after turn, from the second turn to the fifth turn. This was first done with an optimized injection. Then, the current in the septum magnet was changed slightly in order to cause an injection missteering. Figure 25 shows two streak camera images of the bunches observed during the second and the third turn in the case of an injection missteering.

The bunch spacing indeed varies from $50 \mathrm{ps}$ at the second turn to $85 \mathrm{ps}$ at the third turn, whereas it should be constant and equal to 67 ps for a combination factor 5 .

The bunch-to-bunch distance variations were therefore linked to injection errors. An explanation of this effect is given below. The distance between the bunches is closely related to the isochronicity of the ring. Although the isochronicity condition simplifies to a zero momentum compaction $\alpha_{c}$ in the case of achromatic lattices and high-energy electrons, the most general condition for which the time of flight variation between particles vanishes is given by

$$
\left(\frac{1}{L} \int_{L} \frac{C(s)}{\rho(s)} d s\right) x_{0}+\left(\frac{1}{L} \int_{L} \frac{S(s)}{\rho(s)} d s\right) x_{0}^{\prime}+\left(\alpha_{c}-\frac{1}{\gamma^{2}}\right) \frac{\Delta p}{p}=0 \quad \forall x_{0}, x_{0}^{\prime}, \frac{\Delta p}{p},
$$

where $C(s)$ and $S(s)$ are the cosinelike and sinelike solutions of the equation of motion, respectively, and $\rho(s)$ is the bending radius. This condition includes two integrals multiplied by the initial conditions $x_{0}$ and $x_{0}^{\prime}$ of the beam in the horizontal plane. These integrals are in fact the two elements $R_{51}$ and $R_{52}$ of the transfer matrix of the ring evaluated at the observation point for one turn and are

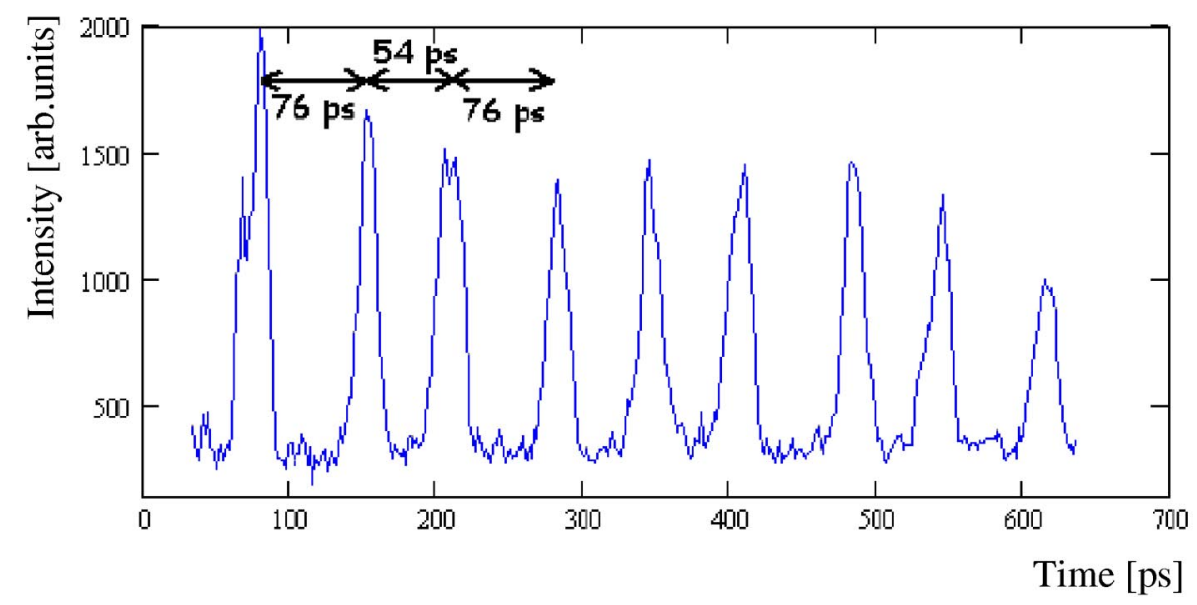

FIG. 24. (Color) Longitudinal intensity profile for bunch train combination of factor 5 showing bunch spacing variations. Here, the correct bunch spacing should be $333 / 5 \simeq 67$ ps. 


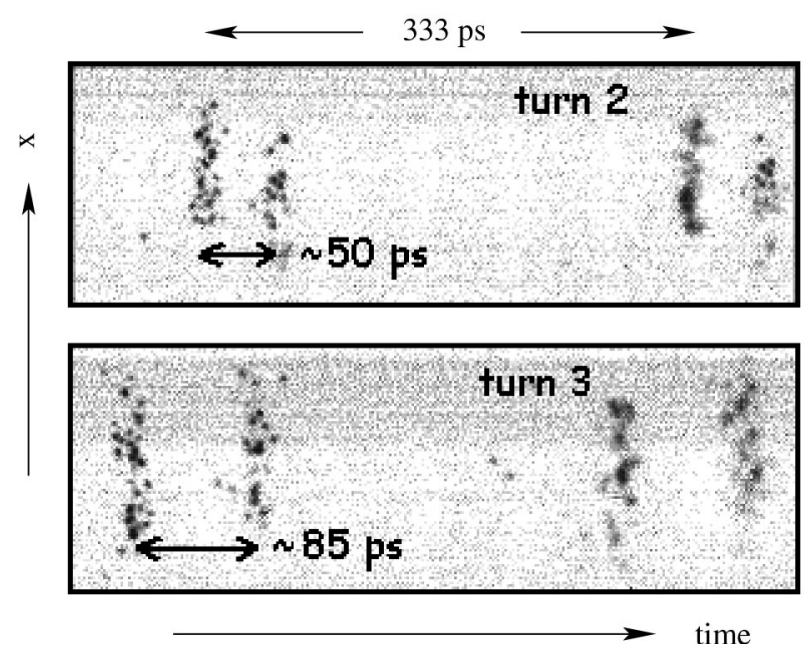

FIG. 25. Turn-to-turn bunch spacing variations during the combination of two pulses with injection errors. The bunch spacing changes from 50 ps at the second turn (top panel) to $85 \mathrm{ps}$ at the third turn (bottom panel).

therefore easily calculated. In the case of achromatic lattices where $D=D^{\prime}=0$ at the observation point, these integrals vanish and the isochronicity is reduced to $\alpha_{c}-$ $1 / \gamma^{2}=0$. In the case where $D \neq 0$ and $D^{\prime} \neq 0$ at the observation point, the integrals evaluated over one turn are not zero.

Indeed, some of our observation points had a nonzero dispersion. During the first combination experiments, the injection process was not optimized, resulting in bunch oscillations around the closed orbit. The orbits were therefore different turn after turn, which implied different conditions $x_{0}$ and $x_{0}^{\prime}$ at the observation point for each turn. According to Eq. (3) with nonzero integrals, the orbit variations were then transformed into variations in time of flight for the particles of the bunches. This delay in the time of flight was visible in the streak camera during the combination, for which subsequent bunches travel a different number of turns.

Figure 26 shows a comparison between the measurements and the model predictions for the bunch spacing observed at two different observation points for large injection errors.

For each turn, 15 streak camera images were recorded, and the error bars on the measured bunch spacing show the extreme measured values.

The solid curves are obtained when using the ring transfer matrix given by the model and by using the initial conditions $x_{0}$ and $x_{0}^{\prime}$ as free parameters to fit the measurements in one of the observation points. For the same initial conditions $\left(x_{0}=0.8 \mathrm{~mm}, x_{0}^{\prime}=0.6 \mathrm{mrad}\right)$, the model predicts exactly the bunch spacing measured at the second location, showing that the mechanism leading to the variable spacing is well understood.

Figure 27 shows a comparison between the measurements and the model predictions for the bunch spacing at one point as a function of the number of turns in the case of small and large injection errors.

The fit parameters are $x_{0}=-0.2 \mathrm{~mm}, x_{0}^{\prime}=0.2 \mathrm{mrad}$ in the case of the optimized injection. Both cases show a very good agreement between the model and the measurements. In addition, the optimized case shows that it is possible to reduce the variations to very low levels ( $2 \mathrm{ps}$ peak to peak, within the measurement resolution) by minimizing the injection errors.

This study shows that the variations of the bunch spacing observed on the streak camera images after the combination are understood and explained by the nonachromatic lattice at the streak camera observation point. By definition, this effect is dependent on the observation

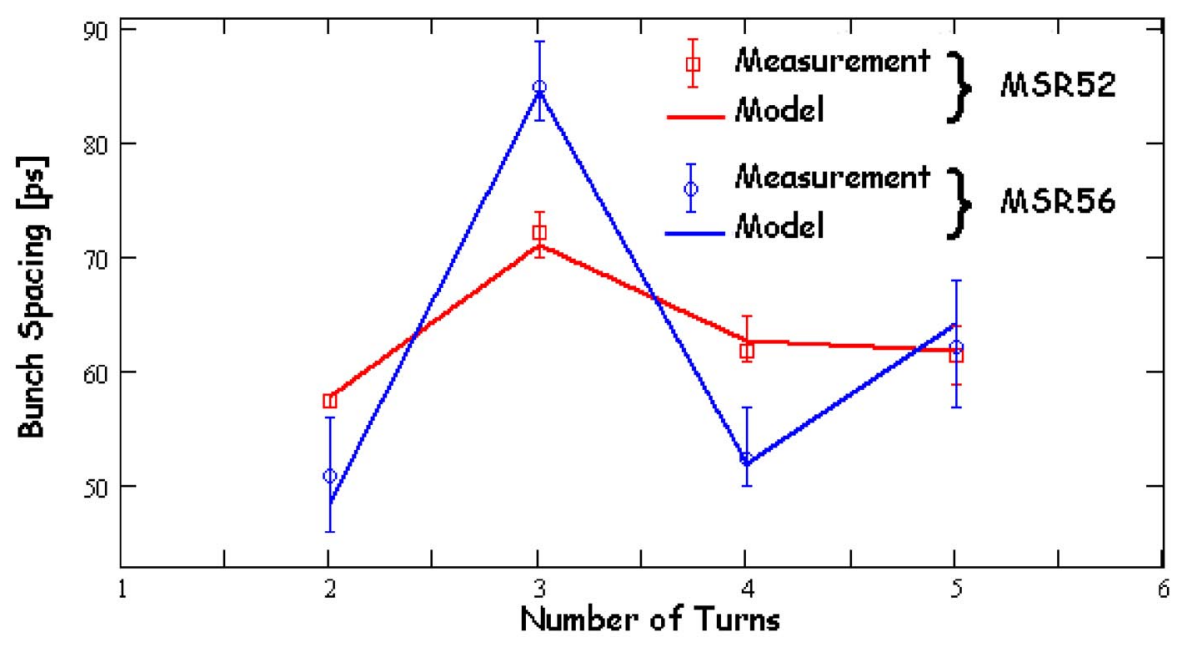

FIG. 26. (Color) Bunch spacing variations after the combination of two pulses as a function of the number of turns observed from two different synchrotron light ports (squares and circles, respectively) in the case of large injection errors. The solid curves correspond to the model predictions in both cases. 


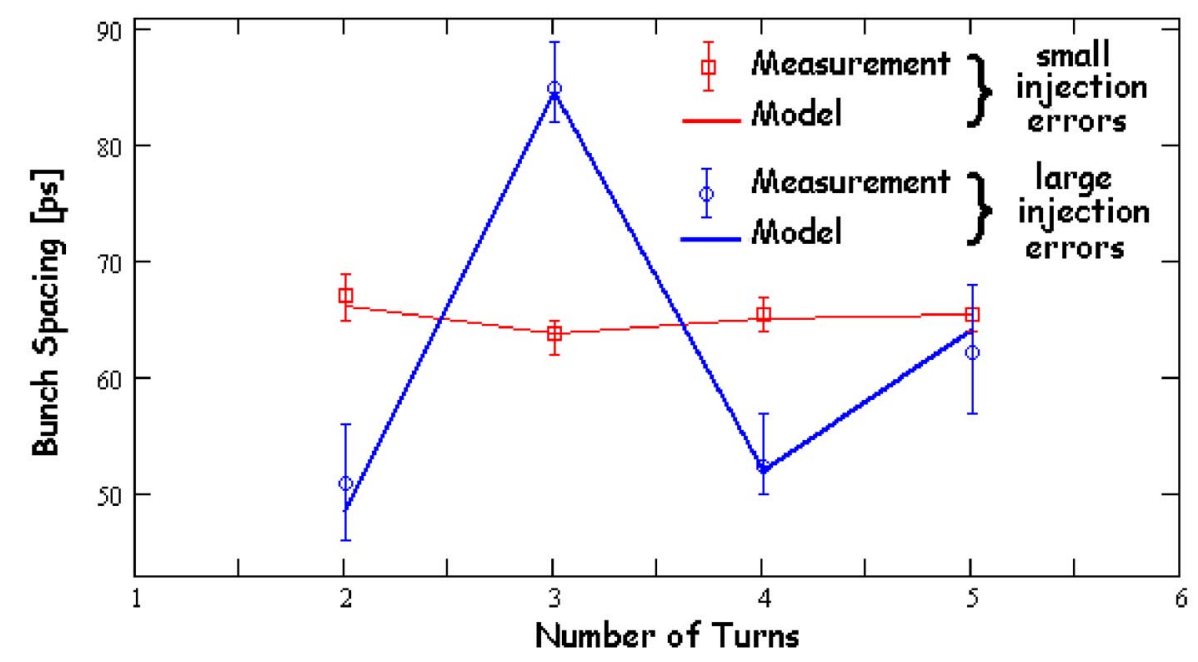

FIG. 27. (Color) Bunch spacing variations after the combination of two pulses as a function of the number of turns for large (circles) and small (squares) injection errors. The solid curves correspond to the model predictions in both cases.

point. In the injection (or extraction) region of the ring, where the achromatic condition is true, the correct bunch spacing is restored in first order, and the effect vanishes. In addition, it has been experimentally demonstrated that this effect can be well controlled already at an observation point with a nonachromatic lattice. Therefore, the variations should be even smaller for the extracted beam, so that this effect does not deteriorate rf power production at all.

\section{Measurements with a bunch frequency monitor}

An alternative method, based on beam frequency spectrum analysis, was used to monitor the frequency multiplication [15]. A coaxial pickup and its readout electronics were designed and mounted in the CTF3 combiner ring in order to allow comparison of the amplitudes of five harmonics of the fundamental beam frequency $(3 \mathrm{GHz})$ while combining the bunch trains.

The signal from this pickup was transported to the readout electronics, amplified and split into five channels with bandpass filters to select the harmonic of interest $(9$, $12,15,18$, and $21 \mathrm{GHz}$ ). Each channel was rectified by a diode used in the square law region to produce the envelope of the rf signal and analyzed with an oscilloscope.

Before making measurements with the beam, a calibration was performed to check the frequency response of the readout electronics and to measure its amplitude response at each frequency of interest. It turned out that the amplitude of the output signal was strongly dependent on the length of the rf pulse at the input of the readout electronics. In particular, for an rf pulse of a few ns (the
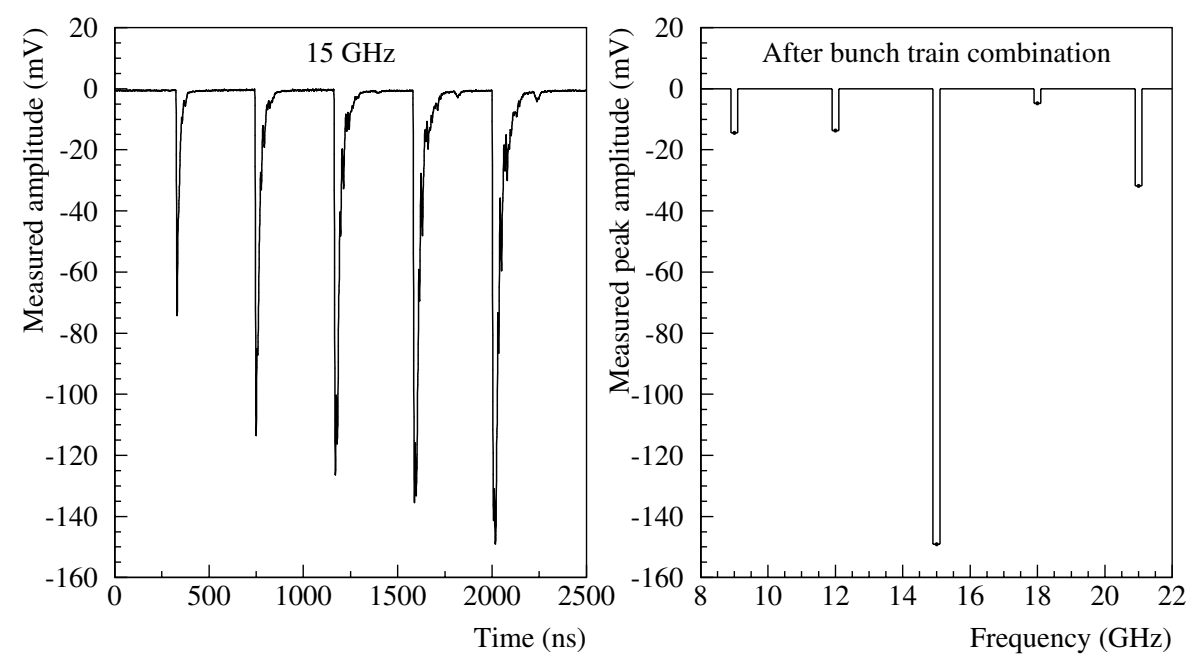

FIG. 28. Bunch frequency monitor signals at $15 \mathrm{GHz}$, while a bunch train combination with a factor 5 occurs (left panel) and amplitude of the five harmonics of interest measured on the oscilloscope at the end of the bunch frequency multiplication (right panel). 

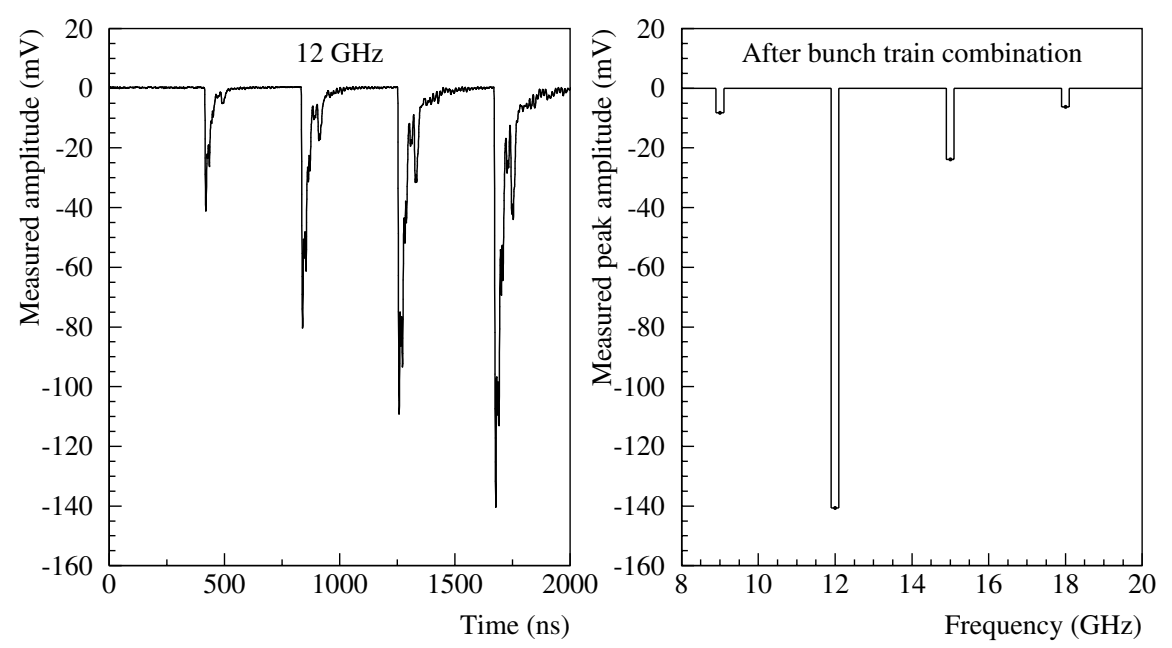

FIG. 29. Bunch frequency monitor signals at $12 \mathrm{GHz}$, while a bunch train combination with a factor 4 occurs (left panel) and amplitude of the four harmonics of interest measured on the oscilloscope at the end of the bunch frequency multiplication (right panel).

length of a bunch train in the CTF3 Preliminary Phase), the readout electronics did not reach a steady state and the peak amplitude of the output signal was thus smaller than in the case of a long input rf pulse. Amplitude fluctuations in the measurements could thus be expected due to the uncertainties on the shape and the length of the bunch trains in the ring.

The commissioning of this bunch frequency monitor with beam was a successful proof of principle. When combining five bunch trains, a clear increase of the $15 \mathrm{GHz}$ signal was observed, see the left plot of Fig. 28, while other harmonics disappear. At the end of the bunch frequency multiplication process, most of the power is found in the $15 \mathrm{GHz}$ harmonic, as shown in the right plot of Fig. 28. Similar results were also obtained for a bunch frequency multiplication with a factor 4; see Fig. 29.

However, a few limitations were also identified during the commissioning of the bunch frequency monitor. In particular, some signal was received by channels at frequencies other than 15 or $12 \mathrm{GHz}$ after the bunch frequency multiplication, with a pattern that is not consistent with a systematic phase error at injection. Also, a few discrepancies were found between the expected and measured signal amplitudes during the bunch train combination. This is probably due to the short length of the bunch trains and to the fact that the longitudinal overlap between the bunch trains is not perfect. Indeed, a time domain analysis of the signal coming from the pickup

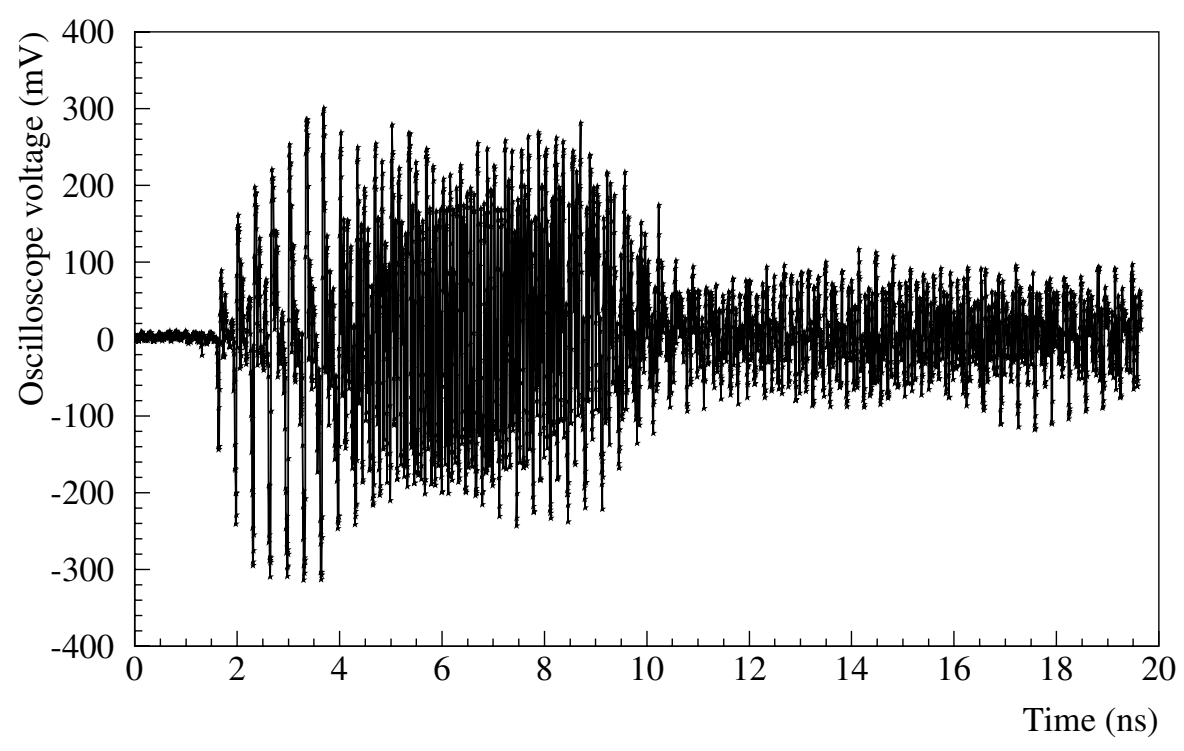

FIG. 30. Time structure of the signal induced in the pickup by the passage of a bunch train in the CTF3 ring after combination. The signal up to $4 \mathrm{~ns}$ is mainly a $3 \mathrm{GHz}$ signal induced by one train; the high frequency signal from the combination is present mainly between 4 and $8 \mathrm{~ns}$. The signals later than $10 \mathrm{~ns}$ are attributed to parasitic waveguide modes. 
showed that the bunch frequency multiplication mainly occurs in the core of the final pulse and not at the edges, because of the pulse-to-pulse variations in length; see Fig. 30. Another reason for these discrepancies may be the presence of parasitic waveguide modes, which can be excited along the beam path and propagate together with the bunch train. Depending on their phase, they can induce either an increase or a decrease of the output signal amplitude. These parasitic modes could also clearly be seen in the time domain measurements.

\section{CONCLUSIONS}

The CTF3 Preliminary Phase has successfully demonstrated the electron beam combination with a multiplication of the bunch repetition frequency and the beam current. It validated the principle of injecting and combining several bunch trains in an isochronous ring using rf deflectors.

After initial commissioning of the installation, optics measurements were performed in the different parts of CTF3 and showed, in general, a good agreement with the expected performance. In particular, streak camera measurements showed that the bunch length could be kept constant over several turns in the isochronous ring and that it was comparable to the one measured at the end of the linac (in the range of $3 \mathrm{ps} \mathrm{rms}$ ). This proved the isochronicity of both ring and injection line, as required for the bunch train combination. Trains of $6 \mathrm{~ns}$ length with a bunch charge of about $0.1 \mathrm{nC}$ were successfully combined with multiplication factors up to 5 and studied in detail for factors 4 and 5. A procedure was then developed to optimize the combination performance, and a combination without measurable losses was established. This procedure minimized simultaneously bunch-to-bunch variations in distance and transverse position, which are well understood and explained. In addition, an alternative instrumentation, based on beam frequency spectrum analysis, was successfully commissioned for monitoring the combination process.

This proof of principle experiment constitutes a crucial step in the CLIC study. In the next stage of CTF3 [16], the bunch train combination will have to be proven at higher bunch charge (2.3 nC) and with longer pulses (140 ns), as required for CLIC.

\section{ACKNOWLEDGMENTS}

The authors would like to thank the laboratories that strongly contributed to the success of CTF3: LAL (Laboratoire de l'Accélerateur Linéaire-Orsay) and, in particular, G. Bienvenu and M. Bernard who designed and built the thermionic gun and its pulser; LNF (Laboratori Nazionale di Frascati) and, in particular, the team of A. Ghigo who designed and implemented the rf deflector and actively participated in the commissioning; Uppsala University (Sweden) and, in particular,
A. Rydberg for the development of the bunch frequency monitor. The CLIC study leader J.P. Delahaye and his deputy I. Wilson and the CTF3 project leader G. Geschonke and his deputy $\mathrm{H}$. Braun provided a full support to transform the former LEP Pre-Injector into a new CLIC Test Facility. Many groups of the former PS Division and several colleagues from the other CERN divisions contributed so strongly and efficiently that without this support the Preliminary Phase of CTF3 would not have been such a big success. The research of A. F. has been supported by a Marie Curie Fellowship of the European Community Programme "Improving Human Research Potential and the Socio-economic Knowledge Base" under Contract No. HPMF-CT-2000-00865.

[1] The CLIC Study Team, G. Guignard, CERN Report No. CERN-2000-008, 2000.

[2] The LEP Injector Study Group, CERN Report No. CERN-LEP/TH/83-29, 1983.

[3] G. Bienvenu, M. Bernard, J. L. Duff, H. Hellgren, R. Pittin, and L. Rinolfi, in Proceedings of the EPAC 2002, Paris, France (EPS-IGA/CERN, Geneva, 2002), p. 1753.

[4] D. Allard et al., CERN Report No. CERN/PS 2001-072 (RF), 2001.

[5] D. Alesini, A. Kucharczyk, S. Kulinski, M. Pachan, E. Plawski, R. Boni, A. Gallo, and F. Marcellini, in Proceedings of the EPAC 2002, Paris, France (Ref. [3]), p. 2115.

[6] R. Corsini, A. Ferrari, L. Rinolfi, T. Risselada, P. Royer, and F. Tecker, CERN CLIC Note No. 470, 2001.

[7] H. Grote and F. Iselin, CERN Report No. CERN SL/ 90-13 (AP), 1990.

[8] R. Corsini and P. Royer, in Proceedings of the EPAC 2002, Paris, France (CLIC Note No. 523, 2002), p. 494.

[9] R. Corsini, B. Dupuy, A. Ferrari, L. Rinolfi, P. Royer, and F. Tecker, in Proceedings of the EPAC 2002, Paris, France (CLIC Note No. 522, 2002), p. 491.

[10] R. Corsini, A. Ferrari, L. Rinolfi, P. Royer, and F. Tecker, CERN PS/AE Note No. 2002-141, 2002.

[11] D. Alesini et al., CERN CTF3 Note No. 044, 2000; CERN PS/AE Note No. 2002-045, 2002.

[12] R. Corsini, A. Ferrari, R. Rinolfi, T. Risselada, P. Royer, and F. Tecker, CERN CTF3 Note No. 009, 2000; CERN PS/LP Note No. 2000-01, 2000.

[13] R. Corsini, A. Ferrari, R. Rinolfi, T. Risselada, P. Royer, and F. Tecker, CERN CTF3 Note No. 013, 2000; CERN PS/LP Note No. 2000-02, 2000.

[14] C. Biscari, R. Corsini, A. Ferrari, A. Gallo, A. Ghigo, L. Rinolfi, P. Royer, and F. Tecker, CERN CTF3 Note No. 054, 2003.

[15] A. Ferrari, F. Caspers, R. Corsini, L. Rinolfi, P. Royer, A. Rydberg, and F. Tecker, in Proceedings of the DIPAC 2003, GSI, Mainz, Germany (GSI, Mainz, 2003), p. 211.

[16] G. Geschonke and A. Ghigo, CERN Report No. CERN/ PS 2002-008 (RF), 2002. 